\title{
MAXIMAL ABELIAN SUBALGEBRAS IN FINITE FACTORS OF TYPE II
}

\author{
BY \\ SISTER RITA JEAN TAUER
}

1. Introduction. The general study of algebras of operators on Hilbert space has led to the investigation of rings of operators, also called $W^{*}$ algebras or von Neumann algebras. These are self-adjoint, weakly closed algebras of operators which contain the identity. If the center of a ring (center in the algebraic sense) consists only of scalar multiples of the identity, then the ring is a factor. Factors have been studied extensively and divided into types by Murray and von Neumann $[5 ; 6]$. In his work on reduction theory [9], von Neumann has considered the decomposition of a ring with respect to various subalgebras contained in its center. When the subalgebra actually is the center, then the rings making up the decomposition are factors. The question of decomposition with respect to a subalgebra which is not the center of the ring, but is maximal abelian in its commutant, is also of interest. Here, each of the rings in the decomposition is isomorphic to the ring of all bounded operators on some Hilbert space [4]. This sort of decomposition is not unique, but rather depends essentially upon the choice of the maximal abelian subalgebra. However, not much is known about these subalgebras, even in the case of a continuous factor of finite type, or a type $\mathrm{II}_{1}$ factor.

In this paper we restrict ourselves to the study of approximately finite $\mathrm{II}_{1}$ factors, that is, those which are generated by a sequence of factors $\mathfrak{M}_{n}$ of type $\mathrm{I}_{n}$, with $\mathfrak{M}_{n_{1}} \varsubsetneqq \mathfrak{M}_{n_{2}} \varsubsetneqq \ldots$. (The factor $\mathfrak{M}_{n}$ is essentially an $n$ by $n$ matrix algebra.) It is proved in $[6, \$ 4.7]$ that all approximately finite factors are algebraically isomorphic, while $[6, \S 1.6]$ shows that the concept of a subring of a finite factor is purely algebraic. This permits one to obtain general results through specific constructions.

Dixmier has defined three types of maximal abelian subalgebras $\mathbf{R}$ in a factor $\mathfrak{A}$, as follows: Let $\mathfrak{R}(\mathbf{R})=\mathbf{P}$ be the ring generated by $\mathscr{V}=\left\{V: V \mathbf{R} V^{*}\right.$ $=\mathbf{R}, V$ unitary, $V \in \mathfrak{U}\}$. Then $\mathbf{R}$ is regular if $\mathbf{P}=\mathfrak{A}, \mathbf{R}$ is semi-regular but not regular if $\mathbf{P}$ is a factor not equal to $\mathfrak{A}$, and $\mathbf{R}$ is singular if $\mathbf{P}=\mathbf{R}$. Dixmier has shown the existence of at least one subalgebra of each type in an approximately finite $\mathrm{II}_{1}$ factor [2]. Later Pukánszky proved the existence of a

Received by the editors August 26, 1963. 
countable infinity of singular subalgebras which cannot be pairwise connected by *automorphisms of the factor [7].

Both Dixmier and Pukánszky used groups and group algebras to construct factors. In this paper we use a more straightforward approach. Let $\mathfrak{M}_{p}$ be the full $2^{p}$ by $2^{p}$ matrix algebra over the complex numbers, and $\left\{{ }^{p} E_{i j}: i, j=0,1, \cdots, 2^{p}-1\right\}$ the matrix units which generate it. Imbed $\mathfrak{M}_{p}$ in $\mathfrak{M}_{p+1}$ by letting ${ }^{p} E_{i j}={ }^{p+1} E_{2 i, 2 j}+{ }^{p+1} E_{2 i+1,2 j+1}$. Then $\bigcup_{p=1}^{\infty} \mathfrak{M}_{p}=$ $\mathfrak{M}$ is a *algebra. If $A=\left|a_{i j}\right|$ is in $\mathfrak{M}_{p}$, then

$$
\text { Trace } A=\operatorname{Tr}(A)=\left(1 / 2^{p}\right) \sum_{i=0}^{2^{p}-1} a_{i i}
$$

Make $\mathfrak{M}$ into a pre-Hilbert space $\mathfrak{W}$ as follows: For $A, B$ in $\mathfrak{M}$, let $(A, B)=$ $\operatorname{Tr}\left(B^{*} A\right)$, and $\sqrt{ }(A, A)=[[A]]$, the Hilbert space norm of $A$. If $A$ is in $\mathfrak{M}, B$ in $\mathfrak{S}$, then $[[A B]] \leqq\|A\|[[B]]$, where $\|A\|$ is a finite number $[8$, p. 77]. So $A$ is a bounded operator on $\mathfrak{W}$ and can be extended to the Hilbert space closure $\mathbb{E}$. Let $\mathfrak{A}$ be the weak closure of $\mathfrak{M}$, or the ring generated by $\mathfrak{M}$.

The trace can be extended to $\mathfrak{A}$ by continuity and has these properties: (1) It is a linear functional. (2) $\operatorname{Tr}(A B)=\operatorname{Tr}(B A)$. (3) If $E$ is a projection, $\operatorname{Tr}(E) \in[0,1]$. It is well known, then, that $\mathfrak{A}$ is a continuous factor of finite type, or a $\mathrm{II}_{1}$ factor $[1 ; 6]$. It also follows directly from the definitions that $\mathfrak{A}$ is approximately finite.

In $\$ 2$ of this paper a simple construction is shown to yield a great variety of maximal abelian subalgebras $\mathbf{R}$ of $\mathfrak{A}$. A regular subalgebra is easily identified in $\S 3$. For the construction of singular and semi-regular but not regular subalgebras, the matrix units are divided into two orthogonal sets. Those in one set generate $\mathbf{P}$, those in the other are in $\mathbf{P}^{\perp}$. After presenting an example of a singular subalgebra in $\$ 4$, the paper proceeds to the construction of an infinite sequence $\left\{\mathbf{R}_{n}\right\}$ of semi-regular but not regular subalgebras. These cannot be pairwise connected by *-automorphisms of $\mathfrak{A}$, since each $\mathbf{R}_{\mathbf{n}}$ has a different length, an invariant which is defined thus: For any subring $\mathbf{D}$ of $\mathfrak{A}, \mathfrak{N}(\mathbf{D})$ is the ring generated by the unitaries which leave $\mathbf{D}$ invariant, and $\mathfrak{N}^{j}(\mathbf{D})=\mathfrak{N}\left[\mathfrak{N}^{j-1}(\mathbf{D})\right]$. Then $\mathbf{R}_{n} \varsubsetneqq \mathfrak{N}\left(\mathbf{R}_{n}\right)=\mathbf{P}_{n} \varsubsetneqq \mathfrak{N}\left(\mathbf{P}_{n}\right) \varsubsetneqq \mathfrak{N}^{2}\left(\mathbf{P}_{n}\right) \varsubsetneqq \cdots \varsubsetneqq \mathfrak{N}^{L}\left(\mathbf{P}_{n}\right)=\mathfrak{A}$, where $L=$ length of $\mathbf{R}_{\mathbf{n}}=n$. However, this invariant does not lead to a complete classification of semi-regular subalgebras, and a counter example is presented in $\$ 6$.

The author would like to express her gratitude to Professor James G. Glimm, her advisor, for the problems and methods he suggested and for the painstaking care with which he surpervised the work of this doctoral thesis. 
2. The construction of maximal abelian subalgebras. The following general facts, which are easily verified by computation, will be useful throughout the rest of the paper.

Lemma 2.1. Suppose $U, A$, and $B$ are in $\mathfrak{A}, U$ unitary. Then [[UA]] $=[[A U]]=[[A]],\left(U^{*} A U, B\right)=\left(A, U B U^{*}\right)$, and $[[A]]=\left[\left[A^{*}\right]\right]$.

We now proceed to the construction of maximal abelian subalgebras of $\mathfrak{A}$. Following [6], we call the topology of $\mathscr{E}$ the metric topology.

2.2. Definitions. Let $\mathbf{Y}_{p}$ be the abelian subalgebra generated by $\left\{{ }^{p} E_{k k}: k=0,1, \cdots, 2^{p}-1\right\}$. Let $\mathbf{E}=\bigcup_{p=1}^{\infty} \mathbf{E}_{p}$, also an abelian subalgebra. Let $\left\{U_{t}: t=1,2, \cdots\right\}$ be a set of self-adjoint unitaries such that: (1) $U_{t}$ $\in \mathfrak{M}_{t}$; (2) $U_{t}$ is zero except for 2 by 2 blocks along the main diagonal. (Note that ${ }^{p} E_{k k}$ commutes with $U_{p+h}$ for $h \geqq 1$.) Define $Y_{t}=U_{1} U_{2} \ldots U_{t}$. For each choice of $\left\{U_{t}: t=1,2, \cdots\right\}$, we construct a subalgebra as follows: Let $\mathbf{S}_{p}$ be the subalgebra generated by $\left\{{ }^{p} \widetilde{E}_{k k}:{ }^{p} E_{k k}\right.$ is a generator of $\mathbf{E}_{p}$ and $\left.{ }^{p} \widetilde{E}_{k k}=Y_{p}{ }^{p} E_{k k} Y_{p}^{*}\right\}$. Let $\mathbf{S}=\bigcup_{p=1}^{\infty} \mathbf{S}_{p}$, also a subalgebra (see Lemma 2.3), and $\mathbf{R}=\mathbf{S}^{-}$. For any $A \in \mathfrak{A}$, define the notation $A^{\lfloor p\rfloor}=Y_{p}^{*} A Y_{p}$ (i.e., $A^{\lfloor p\rfloor}$ $\left.=U_{p} U_{p-1} \ldots U_{1} A U_{1} \ldots U_{p}\right)$.

Lemma 2.3. If $p \geqq q$, then ${ }^{q} \widetilde{E}_{k k}=Y_{p}{ }^{q} E_{k k} Y_{p}^{*}$, so $\mathbf{S}_{q} \subset \mathbf{S}_{p}$. Also, $A^{*|p|}=A^{|p|^{*}}$ for any $A \in \mathfrak{A}$.

Proof. Since $U_{q+1}, \cdots, U_{p}$ commute with ${ }^{q} E_{k k}$,

$$
\begin{aligned}
{ }^{q} \widetilde{E}_{k k} & =U_{1} \ldots U_{q}{ }^{q} E_{k k} U_{q} \ldots U_{1} \\
& =U_{1} \ldots U_{q} \cdots U_{p}{ }^{q} E_{k k} U_{p} \ldots U_{1} \\
& =Y_{p}{ }^{q} E_{k k} Y_{p}^{*} . \\
A^{*|p|} & =Y_{p}^{*} A^{*} Y_{p}=\left(Y_{p}^{*} A Y_{p}\right)^{*}=A^{|p|^{*}} .
\end{aligned}
$$

Lemma 2.4. Suppose $D, \bar{A} \in \mathfrak{M}_{p}$, with

$$
D^{|p|}=\sum_{k} \alpha_{k}^{p} E_{k k} \quad \text { and } \quad \bar{A}^{|p|}=\sum_{j \neq k} \alpha_{j k}^{p} E_{j k} .
$$

Then $D$ is in $\mathbf{S} \subset \mathbf{R}$ and $\bar{A}$ is in $\mathbf{R}^{\perp}$.

Proof. By $\$ 2.2, D=Y_{p} D^{|p|} Y_{p}^{*}=\sum_{k} \alpha_{k} Y_{p}^{p} E_{k k} Y_{p}^{*}$, so $D$ is in $\mathbf{S} \subset \mathbf{R}$.

On the other hand, $\bar{A}^{|p|}$ is orthogonal to ${ }^{q} E_{j j}$ for all $j, q$ (by definition of the inner product). Now suppose ${ }^{q} \widetilde{E}_{j j}$ is a generator of $\mathbf{S}_{q}$. We can assume $q \geqq p$, because $q<p$ implies $\mathbf{S}_{\mathbf{q}} \subset \mathbf{S}_{\mathbf{p}}$. Since $\sum_{k=0}^{2^{p}=-1 p} E_{k k} \bar{A}^{|p| p} E_{k k}=0$, it follows that

$$
\sum_{k=0}^{2^{p}-1}{ }^{p} E_{k k} \bar{A}^{|q| p} E_{k k}=\sum_{k=0}^{2^{p}-1} U_{q} \cdots U_{p+1}^{p} E_{k k} \bar{A}^{|p| p} E_{k k} U_{p+1} \cdots U_{q}=0
$$


So $\bar{A}^{|q|}$ is also orthogonal to ${ }^{q} E_{j j}$. Hence

$$
\left(\bar{A}^{|q|},{ }^{q} E_{j j}\right)=\left(\bar{A}, Y_{q}{ }^{q} E_{j j} Y_{q}^{*}\right)=\left(\bar{A},{ }^{q} \widetilde{E}_{j j}\right)=0
$$

(using Lemma 2.1). Therefore $\bar{A}$ is orthogonal to $\mathbf{S}_{r}$ for all $r$, implying that $\bar{A}$ is orthogonal to $\mathbf{S}$.

Now it is shown in $[6$, p. 728$]$ that weak, strong, and metric closure of a subalgebra of a finite factor coincide. So $A$ is also orthogonal to $\mathbf{R}$, the metric or Hilbert space closure of $\mathbf{S}$ in $\mathfrak{A}$.

Theorem 2.5. Let $\mathbf{S}_{p}, \mathbf{S}$, and $\mathbf{R}$ be defined as in $\$ 2.2$. Then $\mathbf{R}$ is maximal abelian as a subalgebra of $\mathfrak{A}$.

Proof. We show first that $\mathbf{R}$ is abelian. Let ${ }^{p} \widetilde{E}_{k k}$ and ${ }^{q} \widetilde{E}_{j j}$ be projections which generate $\mathbf{S}_{p}$ and $\mathbf{S}_{q}$. Assume $p \geqq q$. Then

$$
\begin{aligned}
{ }^{p} \widetilde{E}_{k k}^{q} \widetilde{E}_{j j} & =\left(Y_{p}^{p} E_{k k} Y_{p}^{*}\right)\left(Y_{p}^{q} E_{j j} Y_{p}^{*}\right) \quad \text { (by Lemma 2.3) } \\
& =Y_{p}^{p} E_{k k}{ }^{q} E_{j j} Y_{p}^{*}=Y_{p}^{q} E_{j j}^{p} E_{k k} Y_{p}^{*} \\
& ={ }^{q} \widetilde{E}_{j j}{ }^{p} \widetilde{E}_{k k} .
\end{aligned}
$$

Since $\mathbf{S}$ is an ascending union of abelian algebras, it is abelian. By the standard argument, so is its weak closure $\mathbf{R}$.

Suppose $A$ is in $\mathscr{A}$, and $A$ commutes with $\mathbf{R}$. There is a sequence $A_{n}$ in $\mathfrak{M}$ which converges strongly to $A$. If $x \in \mathscr{H}$ equals $I$, the identity of $\mathfrak{A}$, then $\left[\left[A_{n} x-A x\right]\right]=\left[\left[A_{n}-A\right]\right] \rightarrow 0$, so $A_{n}$ also converges metrically to $A$. Since $A_{n}$ is in $\mathfrak{M}_{p}$ for some $p$, we can write $A_{n}^{[p]}=D_{n}^{[p]}+\bar{A}_{n}^{[p]}$, where $D_{n}^{|p|}=\sum_{k} \alpha_{k}{ }^{p} E_{k k}$ and $\bar{A}_{n}^{|p|}=\sum_{j \neq k} \alpha_{j k}{ }^{p} E_{j k}$. Then by Lemma $2.10, D_{n}$ is in $\mathbf{S} \subset \mathbf{R}$, and $A_{n}$ is in $\mathbf{R}^{\perp}$. A unitary transformation of $A_{n}^{\lfloor p\rfloor}$ yields $A_{n}=D_{n}$ $+\bar{A}_{n}$.

Assume $\lim _{n}\left[\left[\bar{A}_{n}\right]\right] \neq 0$. Then $\left[\left[\bar{A}_{n}\right]\right]^{2}>3 \epsilon^{2}$ for all $n$ and some $\epsilon>0$. Now $A_{n}$ is Cauchy in the metric topology, so there exists $K(\epsilon)$ such that if $t, m>K(\epsilon),\left[\left[A_{t}-A_{m}\right]\right]=\left[\left[\left(D_{t}-D_{m}\right)+\left(\bar{A}_{t}-\bar{A}_{m}\right)\right]\right]<\epsilon$. But $D_{t}-D_{m}$ is in $\mathbf{R}$, and $\bar{A}_{t}-\bar{A}_{m}$ is in $\mathbf{R}^{\perp}$; and so $\left[\left[\overline{A_{t}}-\bar{A}_{m}\right]\right]<\epsilon$ if $t, m>K(\epsilon)$. Choose $t>K(\epsilon)$ and fix $A_{t}$, say in $\mathfrak{M}_{q}$. Consider this $q$ fixed from here on. Now $A$ commutes with ${ }^{q} \widetilde{E}_{k k}$ for $k=0,1, \cdots, 2^{q}-1$, so that $A^{q} \widetilde{E}_{k k}-{ }^{q} \widetilde{E}_{k k} A$ $=0$. Since $A_{n}$ converges strongly to $A$ and there are only $2^{q}$ projections in question, we can choose $m$ such that $\left[\left[A_{m}{ }^{q} \widetilde{E}_{k k}-{ }^{q} \widetilde{E}_{k k} A_{m}\right]\right]^{2}<\epsilon^{2} / 2^{q}$ for all ${ }^{q} \widetilde{E}_{k k}$, and also $m>K(\epsilon)$. Suppose $A_{m}$ is in $\mathfrak{M}_{r}$, where we can assume $r \geqq q$. By Lemmas 2.3 and 2.1 ,

$$
\begin{aligned}
{\left[\left[A_{m}{ }^{q} \widetilde{E}_{k k}-{ }^{q} \widetilde{E}_{k k} A_{m}\right]\right]^{2} } & =\left[\left[A_{m} Y_{r}^{q} E_{k k} Y_{r}^{*}-Y_{r}^{q} E_{k k} Y_{r}^{*} A_{m}\right]\right]^{2} \\
& =\left[\left[Y_{r}^{*} A_{m} Y_{r}^{q} E_{k k}-{ }^{q} E_{k k} Y_{r}^{*} A_{m} Y_{r}\right]\right]^{2} \\
& =\left[\left[A_{m}^{|r|}{ }^{q} E_{k k}-{ }^{q} E_{k k} A_{m}^{|r|}\right]\right]^{2}<\epsilon^{2} / 2^{q} .
\end{aligned}
$$

Now $D_{m}^{|r|}=\sum_{j} \beta_{j}{ }^{r} E_{j j}$, so $D_{m}^{|r| q} E_{k k}-{ }^{q} E_{k k} D_{m}^{|r|}=0$. Hence 


$$
\left[\left[\bar{A}_{m}^{|r| q} E_{k k}-{ }^{q} E_{k k} \bar{A}_{m}^{|r|}\right]\right]^{2}<\epsilon^{2} / 2^{q} .
$$

Let $P\left(\bar{A}_{m}^{|r|}\right)=\sum_{k=0}^{2^{q}-1 q} E_{k k} \bar{A}_{m}^{|r| q} E_{k k}$. Then

$$
\begin{aligned}
{\left[\left[\bar{A}_{m}^{|r|}-P\left(\bar{A}_{m}^{|r|}\right)\right]\right]^{2} } & \leqq \sum_{k=0}^{2^{q}-1}\left[\left[\bar{A}_{m}^{|r| q} E_{k k}-{ }^{q} E_{k k} \bar{A}_{m}^{|r|}\right]\right]^{2} \\
& \leqq 2^{q}\left(\epsilon^{2} / 2^{q}\right)=\epsilon^{2} .
\end{aligned}
$$

But by Lemma $2.1,\left[\left[\bar{A}_{m}^{|r|}\right]\right]^{2}=\left[\left[\bar{A}_{m}\right]\right]^{2}>3 \epsilon^{2}$, and so we must have $\left[\left[P\left(\bar{A}_{m}^{[r]}\right)\right]\right]^{2}>2 \epsilon^{2}$.

However, $\bar{A}_{t}$ is in $\mathfrak{M}_{q}$, and $\bar{A}_{t}^{|q|}=\sum_{k=0}^{2^{q}-1{ }^{q}{ }^{q}} E_{k k} \bar{A}_{t}^{|q| q} E_{k k}=0$, by definition of $\bar{A}_{t}^{[q]}$. It follows that $P\left(\bar{A}_{t}^{\mid r)}\right)=\sum_{k=0}^{2^{q}-1}{ }^{q} E_{k k} \overline{\bar{A}}_{t}^{[r] q} E_{k k}=0$, as in the proof of Lemma 2.4. Thus we have:

$$
\begin{aligned}
\epsilon^{2} & >\left[\left[\bar{A}_{t}^{|r|}-\bar{A}_{m}^{|r|}\right]\right]^{2} \\
& \geqq\left[\left[\sum_{k=0}^{2^{q}-1} E_{k k}\left(\bar{A}_{t}^{|r|}-\bar{A}_{m}^{|r|}\right)^{q} E_{k k}\right]\right]^{2} \\
& =\left[\left[P\left(\bar{A}_{t}^{|r|}\right)-P\left(\bar{A}_{m}^{|r|}\right)\right]\right]^{2}>2 \epsilon^{2} .
\end{aligned}
$$

But this is a contradiction, and so $\lim _{n}\left[\left[\bar{A}_{n}\right]\right]=0$. Since

$$
\lim _{n}\left[\left[D_{n}+\bar{A}_{n}-A\right]\right]=0 \text {, }
$$

we have $\lim _{n}\left[\left[D_{n}-A\right]\right]=0$, with $D_{n}$ in $\mathbf{S}$.

By assumption, $A$ is in $\mathfrak{A}$. Since it is in the metric closure of $\mathbf{S}$, a subalgebra of a finite factor, it is also in the weak closure of $\mathbf{S}[6$, p. 728]. Therefore $A$ is in $\mathbf{R}$, and so $\mathbf{R}$ is maximal abelian in $\mathcal{A}$.

3. A regular subalgebra. In this section we exhibit a regular maximal abelian subalgebra in the approximately finite factor $\mathscr{A}$.

Lemma 3.1. Let $\mathbf{E}$ be defined as in \$2.2. Let $\mathbf{R}=\mathbf{E}^{-}$. Then $\mathbf{R}$ is maximal abelian.

Proof. Let $U_{t}=I$ for $t=1,2,3, \ldots$, so that $Y_{t}=I$ also. Then Theorem 2.5 applies.

TheOREM 3.2. $\mathbf{R}=\mathbf{E}^{-}$is a regular maximal abelian subalgebra.

Proof. Let ${ }^{p} E_{j k}$ be any matrix unit in $\mathfrak{M}$. Define the unitaries

$$
V_{1}={ }^{p} E_{j k}+{ }^{p} E_{k j}+\sum_{s \neq j, k}{ }^{p} E_{s s} \text { and } V_{2}={ }^{p} E_{j k}-{ }^{p} E_{k j}-\sum_{s \neq j, k}{ }^{p} E_{s s .}
$$

$A$ routine computation shows that $V_{1}$ and $V_{2}$ leave $\mathbf{E}$, hence $\mathbf{R}$, invariant. So $V_{1}$ and $V_{2}$ are among the generators of $\mathfrak{R}(\mathbf{R})=\mathbf{P}$.

Since ${ }^{p} E_{j k}=(1 / 2)\left(V_{1}+V_{2}\right)$, any matrix unit in $\mathfrak{M}$ is contained in $\mathbf{P}$. Hence $\mathfrak{M}^{-}=\mathfrak{U}$ is contained in $\mathbf{P}$, and so $\mathbf{P}=\mathfrak{U}$ and $\mathbf{R}$ is regular. 
4. Singular subalgebras. In this section we establish conditions for a singular maximal abelian subalgebra, and then present an example which satisfies the conditions.

4.1. Definitions. The notation is that of $\$ 2.2$, with the following restrictions on the unitaries $U_{t}$ :

Let

$$
I_{1}=\left[\begin{array}{ll}
1 & 0 \\
0 & 1
\end{array}\right]
$$

and let

$$
B_{1}=\left[\begin{array}{rr}
1 / \sqrt{ } 2 & 1 / \sqrt{ } 2 \\
1 / \sqrt{ } 2 & -1 / \sqrt{ } 2
\end{array}\right]
$$

Then $U_{t}=\left({ }^{t} C_{i j}\right)$, where ${ }^{t} C_{i j}$ is a 2 by 2 matrix,

$$
{ }^{t} C_{i j}= \begin{cases}0 & \text { if } i \neq j, \\ I_{1} \text { or } B_{1} \text { if } i=j .\end{cases}
$$

Let $B_{t}=\left({ }^{t} D_{i j}\right)$, where ${ }^{t} D_{i j}$ is a 2 by 2 matrix,

$$
{ }^{t} D_{i j}=\left\{\begin{array}{l}
0 \text { if } i \neq j, \\
B_{1} \text { if } i=j .
\end{array}\right.
$$

Let $\Gamma$ be an infinite set of positive integers $p_{1}<p_{2}<\ldots$. Assume that if $p \in \Gamma$, then we can divide the matrix units ${ }^{p} E_{j k}$ into two sets as follows: (1) If $U_{p+h}^{p} E_{j k} U_{p+h}={ }^{p} E_{j k}$ for all $h \geqq 1$, then ${ }^{p} E_{j k} \in K_{0}$. (2) If ${ }^{p} E_{i k} \notin K_{0}$, then there exists $d(j, k)(1 \leqq d \leqq p)$ such that $U_{p+d}^{p} E_{j k} U_{p+d}={ }^{p} E_{j k} B_{p+d}$, while $U_{p+h}{ }^{p} E_{j k} U_{p+h}={ }^{p} E_{j k}$ for $1 \leqq h \leqq d-1$.

Also assume that if $q \in \Gamma, q>p$, then ${ }^{p} E_{j k} \in K_{0}$ implies

$$
U_{q} \ldots U_{p+1}^{p} E_{j k} U_{p+1} \ldots U_{q}=\sum \alpha_{r s} \cdot{ }^{q} E_{r s}
$$

with ${ }^{q} E_{r s} \in K_{0}$; and ${ }^{p} E_{j k} \notin K_{0}$ implies

$$
U_{q} \cdots U_{p+1}^{p} E_{j k} U_{p+1} \cdots U_{q}=\sum \alpha_{r s}^{q} E_{r s}
$$

with ${ }^{q} E_{r s} \notin K_{0}$. (Note:

$$
U_{q} \ldots U_{p+1}{ }^{p} E_{j k} U_{p+1} \cdots U_{q}=Y_{q}^{*} Y_{p}^{p} E_{j k} Y_{p}^{*} Y_{q}
$$

will henceforth be abbreviated as $U_{q} \ldots{ }^{p} E_{j k} \ldots U_{q}$.)

Lemma 4.2. Suppose $p \in \Gamma,{ }^{p} E_{j k} \notin K_{0}, d(j, k)=d$. Let $g=2^{d} k+a$ $\left(a=0,1, \cdots\right.$, or $\left.2^{d}-1\right)$, so that ${ }^{p+d} E_{g g} \leqq{ }^{p} E_{k k}$. Then for every $r \geqq 0$, there exist exactly $2^{r+1}$ projections ${ }^{p+d+r} E_{h h} \leqq{ }^{p} E_{j j}$ such that

$$
\left[\left[{ }^{p+d+r} E_{h h}\left(U_{p+d+r} \cdots{ }^{p} E_{j k} \ldots U_{p+d+r}\right){ }^{p+d} E_{g g}\right]\right]^{2}=1 / 2^{p+d+r+1} .
$$

Proof. Consider $g=2^{d} k+a$ fixed throughout the proof.

(i) By using $\$ 4.1$, we have: 


$$
\begin{aligned}
& { }^{p+d} E_{h h} U_{p+d} \cdots{ }^{p} E_{j k} \cdots U_{p+d}{ }^{p+d} E_{g g}={ }^{p+d} E_{h h}{ }^{p} E_{j k} B_{p+d}{ }^{p+d} E_{g g} \\
& =\left\{\begin{array}{cl}
1 / \sqrt{ } 2^{p+d} E_{h g} & \text { if } a \equiv 0(\bmod 2) \text { and } h=h(g)=2^{d} j+a \text { or } 2^{d} j+a+1, \\
\pm 1 / \sqrt{ } 2^{p+d} E_{h g} & \text { if } a \equiv 1(\bmod 2) \text { and } h=h(g)=2^{d} j+a-1 \text { or } 2^{d} j+a, \\
0 & \text { otherwise. }
\end{array}\right.
\end{aligned}
$$

Hence the lemma holds for $r=0$.

(ii) Take $r \geqq 1$ and assume that

$$
\begin{aligned}
& {\left[\left[{ }^{p+d+r-1} E_{h h}\left(U_{p+d+r-1} \cdots{ }^{p} E_{j k} \cdots U_{p+d+r-1}\right)^{p+d} E_{g g}\right]\right]^{2}} \\
& \quad= \begin{cases}1 / 2^{p+d+r} & \text { if }{ }^{p+d+r-1} E_{h h} \leqq{ }^{p+d} E_{h(g), h(g)}, \\
0 & \text { otherwise. }\end{cases}
\end{aligned}
$$

Let

$$
p+d+r=s, \quad{ }^{s-1} E_{h h}=\sum_{i=1}^{2}{ }^{s} E_{h_{i} h_{i}}, \quad{ }^{p+d} E_{g g}=\sum_{\nu=1}^{2 r-1}{ }^{s-1} E_{g_{v} g_{\nu}} .
$$

Then ${ }^{s-1} E_{h h}\left(U_{s-1} \ldots{ }^{p} E_{j k} \ldots U_{s-1}\right){ }^{s-1} E_{g_{\nu} g_{\nu}}$ is a multiple (possibly zero) of a matrix unit ${ }^{s-1} E_{h g_{v}}$. Because of the form of $U_{s}$,

$$
\begin{aligned}
& {\left[\left[{ }^{s} E_{h_{1} h_{1}} U_{s}\left(U_{s-1} \ldots{ }^{p} E_{j k} \cdots U_{s-1}\right) U_{s}{ }^{s-1} E_{g_{\nu} g_{\nu}}\right]\right]^{2}} \\
& =\left[\left[{ }^{s} E_{h_{2} h_{2}} U_{s}\left(U_{s-1} \cdots{ }^{p} E_{j k} \ldots U_{s-1}\right) U_{s}{ }^{s-1} E_{g_{\nu b_{\nu}}}\right]\right]^{2} .
\end{aligned}
$$

So the sums over $\nu$ of these two expressions are equal. Since the norms are preserved by the unitaries $U_{s}$, we have:

$$
\begin{aligned}
\sum_{\nu=1}^{2 r-1}\left[\left[{ }^{s} E_{h_{i} h_{i}} U_{s} \ldots{ }^{p} E_{j k} \ldots U_{s}{ }^{s-1} E_{g, g_{\nu}}\right]\right]^{2} \\
=(1 / 2) \sum_{\nu=1}^{2 r-1}\left[\left[{ }^{s-1} E_{h h} U_{s} \ldots{ }^{p} E_{j k} \ldots U_{s}^{s-1} E_{g_{\nu} g_{\nu}}\right]\right]^{2} \\
=(1 / 2)\left[\left[{ }^{s-1} E_{h h} U_{s-1} \ldots{ }^{p} E_{j k} \ldots U_{s-1}{ }^{p+d} E_{g g}\right]\right]^{2} \\
= \begin{cases}1 / 2^{s+1} & \text { if }{ }^{s-1} E_{h h} \leqq{ }^{p+d} E_{h(g), h(g)}, \\
0 & \text { otherwise. }\end{cases}
\end{aligned}
$$

Hence $\left[\left[{ }^{p+d+r} E_{h_{i} h_{i}}\left(U_{s} \cdots{ }^{p} E_{j k} \ldots U_{s}\right)^{p+d} E_{g g}\right]\right]^{2}$ equals $1 / 2^{p+d+r+1}$ if and only if ${ }^{s} E_{h_{i} h_{i}}={ }^{p+d+r} E_{h_{i} h_{i}} \leqq{ }^{p+d} E_{h(g), h(g)}$, or for $2\left(2^{\prime}\right)$ projections ${ }^{p+d+r} E_{h_{i} h_{i}}$.

Lemma 4.3. Suppose $\mathbf{R}$ is constructed according to $\$ 2.2$ and $\$ 4.1$, and $\mathscr{V}=\left\{V: V \mathbf{R} V^{*}=\mathbf{R}, V\right.$ unitary, $\left.V \in \mathfrak{A}\right\}$. If $V \in \mathscr{V}$, then there is a sequence $V_{n}$ converging metrically to $V$, such that if $V_{n} \in \mathfrak{M}_{p}(p \in \Gamma)$, then $V_{n}^{|p|}$ $=\sum \beta_{a b}{ }^{p} E_{a b}$ with ${ }^{p} E_{a b} \in K_{0}$.

Proof. Let $V$ be in $\mathscr{V}$. Then there is a sequence $W_{n} \in \mathfrak{M}$ which converges strongly to $V$. Since $W_{n} \in \mathfrak{M}$, we must have $W_{n} \in \mathbb{M}_{p}$ for some $p \in \Gamma$, 
the set referred to in $\$ 4.1$. Then we can write $W_{n}^{\lfloor p\rfloor}=V_{n}^{\lfloor p\rfloor}+\bar{W}_{n}^{|p|}$, where $V_{n}^{[p]}=\sum \beta_{a b}{ }^{p} E_{a b}$ with ${ }^{p} E_{a b} \in K_{0}$ and $\bar{W}_{n}^{\mid p]}=\sum \alpha_{j k}{ }^{p} E_{j k}$ with ${ }^{p} E_{j k} \notin K_{0}$. By the last paragraph of $\$ 4.1$, this decomposition is independent of $p$, i.e., if $q>p, q \in \Gamma$, then

$$
V_{n}^{|q|}=U_{q} \ldots U_{p+1} V_{n}^{|p|} U_{p+1} \cdots U_{q}
$$

and

$$
\bar{W}_{n}^{|q|}=U_{q} \ldots U_{p+1} \bar{W}_{n}^{|p|} U_{p+1} \cdots U_{q} .
$$

So $W_{n}=V_{n}+\bar{W}_{n}$, and $\left(V_{n}, \bar{W}_{n}\right)=0$.

Suppose $\lim _{n}\left[\left[\bar{W}_{n}\right]\right] \neq 0$. Then $\left[\left[\bar{W}_{n}\right]\right]^{2}>16 \epsilon^{2}$ for all $n$ and some $\epsilon>0$. We can assume $\epsilon \leqq 1 / 9$. Since $W_{n}$ is Cauchy in the strong and metric topologies and $\left(V_{n}, \bar{W}_{n}\right)=0$, there exists $K(\epsilon)$ such that if $m, t>K(\epsilon)$, then $\left[\left[\bar{W}_{t}-\bar{W}_{m}\right]\right]<\epsilon$. Choose $t>K(\epsilon)$ and fix $W_{t}$. If $W_{t} \in \mathfrak{M}_{p}(p \in \Gamma)$, then $\bar{W}_{t}^{\mid p\rfloor}=\sum \alpha_{j k}{ }^{p} E_{j k}\left({ }^{p} E_{j k} \notin K_{0}\right)$ implies that

$$
\left(1 / 2^{p}\right) \sum\left|\alpha_{j k}\right|^{2}>16 \epsilon^{2} \text {. }
$$

Consider $p$ fixed from here on. Next choose $m>K(\epsilon)$ and also such that $\left[\left[W_{m}-V\right]\right]<\epsilon / 32\left(2^{2 p}\right)$.

For now, fix one pair $(j, k)$ in the sum for $\bar{W}_{t}^{\mid p\rfloor}$, and let $d=d(j, k)$, as in \$4.1. Consider the $2^{d}$ projections $E_{g}$ such that $E_{g}^{\lfloor p+d]}={ }^{p+d} E_{g g} \leqq{ }^{p} E_{k k}$. By definition, $E_{g} \in \mathbf{R}$, and so $V E_{g} V^{*}=F_{g} \in \mathbf{R}$, with $F_{g}$ a projection. Since $V E_{g}-F_{g} V=0,\left[\left[W_{m} E_{g}-F_{g} W_{m}\right]\right]<\epsilon / 16\left(2^{2 p}\right)$. By [6, p. 761], there is a sequence of projections $F_{g_{i}} \in \mathrm{S}$ (see $\left.\$ 2.2\right)$ such that $\left[\left[F_{g_{i}}-F_{g}\right]\right] \rightarrow 0$. Choose $i$ (for each $g$ ) such that $\left[\left[W_{m} E_{g}-F_{g_{i}} W_{m}\right]\right]<\epsilon / 8\left(2^{2 p}\right)$. Let $r=r(j, k$ ) be large enough so that $W_{m}$ and all $F_{g_{i}}$ are in $\mathfrak{M}_{p+d+r}$, and so that $p+d+r$ $\in \Gamma$. Let $s=s(j, k)=p+d+r$. Then

$$
\begin{aligned}
{\left[\left[W_{m}^{|s|} E_{g}^{|s|}-F_{g_{i}}^{\mid s]} W_{m}^{|s|}\right]\right] } & =\left[\left[\left(V_{m}^{|s|} E_{g}^{|s|}-F_{g_{i}}^{\mid s]} V_{m}^{\mid s\}}\right)+\left(\bar{W}_{m}^{|s|} E_{g}^{|s|}-F_{g_{i}}^{\mid s\rfloor} \bar{W}_{m}^{\mid s\}}\right)\right]\right] \\
& <\epsilon / 8\left(2^{2 p}\right) .
\end{aligned}
$$

Since $E_{g}$ and $F_{g_{i}}$ are in $\mathbf{R} \cap \mathfrak{M}_{s}, E_{g}^{|s|}$ and $F_{g_{i}}^{|s|}$ are sums of minimal projections ${ }^{s} E_{\beta \beta}$. Hence by definition of $V_{m}^{|s|}$ and $\vec{W}_{m}^{|s|}$, the two expressions in parentheses are orthogonal, and so

$$
\left[\left[\bar{W}_{m}^{|s|} E_{g}^{|s|}-F_{g_{i}}^{\mid s]} \bar{W}_{m}^{|s|}\right]\right]<\epsilon / 8\left(2^{2 p}\right) .
$$

Suppose $F_{g_{i}}^{|s|}=\sum^{s} E_{\beta(g), \beta(g)}$. Let $G_{g}=\{h: h \neq \beta(g)$ for any $\beta(g)$ in the sum, and $\left.{ }^{s} E_{h h} \leqq{ }^{p} E_{j j}\right\}$. Recall that $E_{g}^{\mid s]}=U_{s} \ldots{ }^{p+d} E_{g g} \ldots U_{s}={ }^{p+d} E_{g g}$. Then

$$
\sum_{G_{g}}\left[\left[{ }^{s} E_{h h} \bar{W}_{m}^{[s] p+d} E_{g g}\right]\right]^{2}\left[=\left[\left[\bar{W}_{m}^{[s] p+d} E_{g g}-F_{g i}^{[s]} \bar{W}_{m}^{[s]}\right]\right]^{2}<\epsilon^{2} / 64\left(2^{4 p}\right) .\right.
$$

On the other hand, let us consider ${ }^{p} E_{j j} \bar{W}_{t}^{[s] p} E_{k k}=\alpha_{j k} U_{s} \cdots{ }^{p} E_{j k} \cdots U_{s}$. Now ${ }^{p} E_{j k} \notin K_{0}, s=p+d+r$, and ${ }^{p+d} E_{g g} \leqq{ }^{p} E_{k k}$. So Lemma 4.2 asserts that there are exactly $2^{r+1}$ projections ${ }^{s} E_{h h} \leqq{ }^{p} E_{j j}$ such that 


$$
\begin{aligned}
& {\left[\left[{ }^{s} E_{h h} \bar{W}_{t}^{[s]}{ }^{p+d} E_{g g}\right]\right]^{2}} \\
& \quad=\left[\left[\alpha_{j k}{ }^{s} E_{h h} U_{s} \ldots{ }^{p} E_{j k} \ldots U_{s}^{p+d} E_{g g}\right]\right]^{2}=\left|\alpha_{j k}\right|^{2} / 2^{s+1} .
\end{aligned}
$$

Let $H_{g}=\left\{h:{ }^{s} E_{h h} \leqq{ }^{p} E_{j j}\right.$ and the preceding equality holds $\}$. Clearly, $\#\left(H_{g}\right)=2^{r+1}$.

The set we are interested in is $H_{g} \cap G_{g}$. Since $\left[\left[W_{m}-V\right]\right]<\epsilon / 16\left(2^{2 p}\right)$, a routine calculation shows that $\left[\left[V E_{g}-F_{g_{i}} V\right]\right]=\left[\left[V E_{g} V^{*}-F_{g_{i}}\right]\right]$ $<\epsilon / 4\left(2^{2 p}\right)<\epsilon / 4\left(2^{p+d}\right)$. Now $V$ is unitary, $\left[\left[E_{g}\right]\right]=\sqrt{ }\left(1 / 2^{p+d}\right)$, and $\epsilon \leqq 1 / 9$, so it follows that $\left[\left[F_{g_{i}}\right]\right]^{2}=\left[\left[\sum^{p+d+r} E_{\beta(g), \beta(g)}\right]\right]^{2}<17 / 16\left(2^{p+d}\right)$. Hence the sum has fewer than $17\left(2^{7}\right) / 16$ terms. But if ${ }^{p+d+r} E_{h h} \leqq{ }^{p} E_{j j}$ and $h \neq \beta(g)$, then $h \in G_{g}$. Therefore,

$$
\begin{aligned}
\#\left(H_{g} \cap G_{g}\right) & \geqq \#\left(H_{g}\right)-\#\left\{\beta(g):{ }^{s} E_{\beta(g), \beta(g)} \leqq F_{g i}^{\mid s}\right\} \\
& >2^{r+1}-17\left(2^{r}\right) / 16=15\left(2^{2}\right) / 16 .
\end{aligned}
$$

Let $J=J(j, k)=\left\{(h, g):{ }^{p+d} E_{g g} \leqq{ }^{p} E_{k k}\right.$ and $\left.h \in H_{g} \cap G_{g}\right\}$. Then \#(J) $\geqq 2^{d}\left[\min _{g} \#\left(H_{g} \cap G_{g}\right)\right] \geqq 15\left(2^{d+\eta}\right) / 16$. So

$$
\sum_{J}\left[\left[{ }^{s} E_{h h} \bar{W}_{t}^{[s] p+d} E_{g g}\right]\right]^{2} \geqq 15\left|\alpha_{j k}\right|^{2} / 32\left(2^{p}\right) .
$$

(We use (3) since $(h, g) \in J$ implies $h \in H_{g}$ for some g.) On the other hand,

$$
\begin{aligned}
\sum_{J}\left[\left[{ }^{s} E_{h h} \bar{W}_{m}^{|s| p+d} E_{g g}\right]\right]^{2} & \leqq 2^{d}\left[\max _{g} \sum_{H_{g} \cap G_{g}}\left[\left[{ }^{s} E_{h h} \bar{W}_{m}^{|s| p+d} E_{g g}\right]\right]^{2}\right] \\
& \left.<\epsilon^{2} / 64\left(2^{2 p}\right) \quad \text { (using (2) and } d \leqq p\right) .
\end{aligned}
$$

From here on, we do not regard $(j, k)$ as fixed, but let $Z=\left\{(j, k):{ }^{p} E_{j k}\right.$ is in the sum for $\left.\bar{W}_{t}^{[p]}\right\}$. Note that $d, r, s$, and $J$ are still functions of $(j, k)$. Let $s_{0}=\max _{Z}[s]$. Then

$$
\begin{aligned}
& \left.\epsilon^{2} \geqq\left[\left[\bar{W}_{t}^{\left[s_{0}\right]}-\bar{W}_{m}^{\left[s_{0}\right.}\right]\right]\right]^{2} \\
& \geqq \sum_{Z}\left[\left[{ }^{p} E_{j j}\left(\bar{W}_{t}^{\left.\mid s_{0}\right]}-\bar{W}_{m}^{\left[s_{0}\right.}\right)^{p} E_{k k}\right]\right]^{2} \\
& =\sum_{Z}\left[\left[{ }^{p} E_{j j}\left(\bar{W}_{t}^{[s]}-\bar{W}_{m}^{|s|}\right){ }^{p} E_{k k}\right]\right]^{2} \\
& \geqq\left\lceil\left[\sum_{Z}\left(\sum_{(h, g) \in J}{ }^{s} E_{h h}\left(\bar{W}_{t}^{[s]}-\bar{W}_{m}^{|s\rangle}\right)^{p+d} E_{g g}\right)||^{2}\right.\right. \\
& \left.\geqq||\left[\sum_{Z} \sum_{J}^{s} E_{h h} \bar{W}_{t}^{|s| p+d} E_{g g}\right] \mid-\left\lceil\mid \sum_{Z} \sum_{J}^{s} E_{h h} \bar{W}_{m}^{|s| p+d} E_{g g}\right]\right]\left.\right|^{2}
\end{aligned}
$$

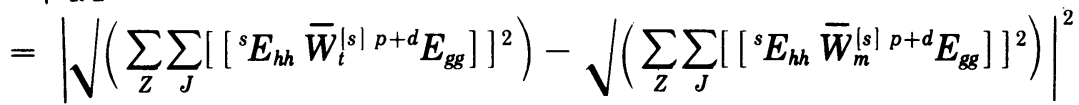

$$
\begin{aligned}
& >\left|\sqrt{ }\left(\sum_{Z} 15\left|\alpha_{j k}\right|^{2} / 32\left(2^{p}\right)\right)-\sqrt{ }\left(\sum_{Z} \epsilon^{2} / 64\left(2^{2 p}\right)\right)\right|^{2} \\
& >\left|\sqrt{ }\left(15\left(16 \epsilon^{2}\right) / 32\right)-\sqrt{ }\left(2^{2 p} \epsilon^{2} / 64\left(2^{2 p}\right)\right)\right|^{2}>\epsilon^{2} \quad(\text { by (1)). }
\end{aligned}
$$


But this is a contradiction. Hence $\lim _{n}\left[\left[\bar{W}_{n}\right]\right]=0$ and $\lim _{n}\left[\left[V_{n}-V\right]\right]$ $=0$, where $V_{n}$ has the required form.

THEOREM 4.4. Let $\mathbf{R}$ be such that the conditions of $\$ 4.1$ are satisfied, and also ${ }^{p} E_{j k} \in K_{0}$ if and only if $j=k$. Then $\mathbf{R}$ is a singular maximal abelian subalgebra.

Proof. As usual, $\mathscr{V}=\left\{V: V \mathbf{R} V^{*}=\mathbf{R}, V\right.$ unitary, $\left.V \in \mathscr{A}\right\}$. Using Lemma 4.3 and the fact that the strong and metric closures of $S$ in $\mathscr{A}$ coincide, we have $\mathscr{V} \subset \mathbf{R}$, and so $\mathbf{P}$, the ring generated by $\mathscr{V}$, is contained in $\mathbf{R}$. On the other hand, any ring is generated by its unitaries $[1$, p. 4], and any unitary in $\mathbf{R}$ is in $\mathscr{V}$, so $\mathbf{R} \subset \mathbf{P}$. Therefore $\mathbf{R}=\mathbf{P}$, and so $\mathbf{R}$ is singular.

4.5. Definitions. In order to construct a subalgebra satisfying the conditions of Theorem 4.4, we define the set $\left\{U_{n}: n=1,2, \cdots\right\}$ as follows: We shall say that $U_{n}$ is of type $r$ (where $r<n$ ) if and only if

$$
{ }^{r} E_{i i} U_{n}=U_{n}{ }^{r} E_{i i}= \begin{cases}{ }^{r} E_{i i} & \text { when } i \equiv 0(\bmod 2), \\ { }^{r} E_{i i} B_{n} & \text { when } i \equiv 1(\bmod 2) .\end{cases}
$$

This completely specifies any $U_{n}$ if we assign to $U_{n}$ an integer $r<n$ to designate its type.

If $n>1$, let $n=2^{s}+r$, where $s=0,1,2, \cdots$ and $1 \leqq r \leqq 2^{s}$. Then we require that $U_{n}$ be of type $r$. We set $U_{1}=I$, and let

$$
\Gamma=\left\{p: p=2^{t}, t=0,1, \cdots\right\} \text {. }
$$

THEOREM 4.6. If $\mathbf{R}$ is constructed according to $\$ 4.5$, then $\mathbf{R}$ is a singular maximal abelian subalgebra.

Proof. Let ${ }^{p} E_{j k}$ be any matrix unit with $p \in \Gamma, p=2^{s}$ for some fixed $s$. If $n>p$, write $n=2^{l}+r$, where $l \geqq s$ and $1 \leqq r \leqq 2^{l}$. First consider the case $r \leqq 2^{s}=p$. By definition, $U_{n}$ is of type $r$. If ${ }^{p} E_{j j} \leqq{ }^{r} E_{u u}$ and ${ }^{p} E_{k k} \leqq{ }^{r} E_{w}$, then

$$
U_{n}^{p} E_{j k} U_{n}=U_{n}^{r} E_{u u}^{p} E_{j k}^{r} E_{v v} U_{n}= \begin{cases}{ }^{p} E_{j k} & \text { if } u \equiv v(\bmod 2), \\ { }^{p} E_{j k} B_{n} & \text { if } u \neq v(\bmod 2) .\end{cases}
$$

Next suppose $r>p$. Then ${ }^{p} E_{j k}=\sum_{i}{ }^{r} E_{j k_{i}}$, where $j_{i}=2^{r-p} j+i$ and $k_{i}=2^{r-p} k$ $+i$, so that $j_{i} \equiv k_{i}(\bmod 2)$ for all $i$. Hence $U_{n}{ }^{p} E_{j k} U_{n}=U_{n} \sum_{i}{ }^{r} E_{j i k_{i}} U_{n}$ $=\sum_{i}{ }^{r} E_{j i k_{i}}={ }^{p} E_{j k}$. So if $n>p, U_{n}{ }^{p} E_{j k} U_{n}$ is equal either to ${ }^{p} E_{j k}$ or to ${ }^{p} E_{j k} B_{n}$. Straightforward calculation shows that if $n>p$, then $U_{n}^{p} E_{j j} U_{n}$ equals ${ }^{p} E_{j j}$, so that ${ }^{p} E_{j j} \in K_{0}$. It follows directly that if $q \in \Gamma, q>p$, then $U_{q} \ldots{ }^{p} E_{j j}$ $\ldots U_{q}=\sum_{i}{ }^{q} E_{j i j i}$, where ${ }^{q} E_{j j_{i}} \in K_{0}$.

Suppose ${ }^{p} E_{j k}$ is such that $j \neq k$. Then there exists $t=0,1, \cdots$, or $p-1$ such that $j \not \equiv k\left(\bmod 2^{t+1}\right)$, while $j \equiv k\left(\bmod 2^{\imath}\right)$. Hence $j=2^{t} j_{0}+i$ and $k=2^{t} k_{0}+i$ for some $j_{0}, k_{0}$ such that $j_{0} \not \equiv k_{0}(\bmod 2)$. If $p=2^{s}$, take $m=2^{s}+(p-t)$. Clearly $1 \leqq p-t \leqq 2^{s}$, so $U_{m}$ is of type $p-t$. Then $U_{m}^{p} E_{j k} U_{m}=U_{m}^{p-t} E_{j j_{0}}^{p} E_{j k}^{p-t} E_{k_{0} k_{0}} U_{m}={ }^{p} E_{j k} B_{m}$, so ${ }^{p} E_{j k} \notin K_{0}$. 
Let $d$ be the smallest positive integer $d^{\prime}$ with the property that $U_{p+d^{\prime}}$ $\times{ }^{p} E_{j k} U_{p+d^{\prime}}={ }^{p} E_{j k} B_{p+d^{\prime}}$. Since $p+1 \leqq p+d \leqq m$, it follows that $1 \leqq d$ $\leqq m-p=p-t \leqq p$. Also, if $1 \leqq h \leqq d-1, U_{p+h}{ }^{p} E_{j k} U_{p+h}$ is not equal to ${ }^{p} E_{j k} B_{p+h}$, so must equal ${ }^{p} E_{j k}$.

The preceding shows that ${ }^{p} E_{j k} \in K_{0}$ if and only if $j=k$. Also, we see that if $j \neq k$, then the alternate condition (i.e., condition (2)) of $\$ 4.1$ is satisfied. If $q \in \Gamma, q>p$, then $j \neq k$ implies $U_{q} \cdots{ }^{p} E_{j k} \cdots U_{q}=\sum \alpha_{r s}{ }^{q} E_{r s}$ with $r \neq s$, so that ${ }^{q} E_{r s} \notin K_{0}$. Thus the final condition of $\$ 4.1$ is verified.

Therefore Theorem 4.4 applies and $\mathbf{R}$ is singular.

5. An infinite sequence of semi-regular subalgebras. In this section we construct an infinite sequence of semi-regular subalgebras which cannot be pairwise connected by ${ }^{*}$-automorphisms of $\mathfrak{A}$.

5.1. Definitions. Throughout this section we regard $n=1,2,3, \cdots$ as fixed. Let $\Gamma=\{p: p=(2 c+1) n, c=0,1,2, \cdots\}$, an infinite set of positive integers. If ${ }^{p} E_{i k}$ is such that $p \in \Gamma$, then ${ }^{p} E_{i k}$ is in some set $K_{\gamma}$, where $\gamma=0$, $1, \cdots$, or $2^{n}-1$. Let $\mathscr{C}_{n}$ be the class containing all the sets $K_{\gamma}$.

Let $\mathcal{S}_{n}$ be the set of all $n$-tuples $\left(a_{1}, a_{2}, \cdots, a_{n}\right)$, where $a_{k}=0$ or 1 . This is a commutative group under the operation of coordinate-wise addition $(\bmod 2)$. Define a function $\phi$ from $\mathscr{C}_{n}$ to $\mathscr{S}_{n}$ as follows: If $\gamma=\sum_{k=1}^{n} a_{k} 2^{n-k}$, then $\phi\left(K_{\gamma}\right)=\left(a_{1}, \cdots, a_{k}, \cdots, a_{n}\right)$. If we define an operation $\otimes$ on $\mathscr{C}_{n}$ by the rule that $K_{\alpha} \otimes K_{\beta}=K_{\delta}$ if and only if $\phi\left(K_{\alpha}\right)+\phi\left(K_{\beta}\right)=\phi\left(K_{\delta}\right)$, then $\phi$ is an isomorphism of $\mathscr{C}_{n}$ onto $\mathscr{S}_{n}$ (with respect to these two operations).

We determine the set $K_{\gamma}$ in which ${ }^{p} E_{i k}$ is contained as follows: For any index $i\left(0 \leqq i \leqq 2^{(2 c+1) n}-1\right)$, let $i=\sum_{r=0}^{2 c} i_{r} 2^{r n}$, an expansion to the base $2^{n}$. Then $0 \leqq i_{r} \leqq 2^{n}-1$, so that $i_{r}=\sum_{k=1}^{n} r_{k} 2^{n-k}$ and $\left(r_{1}, \cdots, r_{k}, \cdots, r_{n}\right)$ $\in \mathbb{S}_{n}$. Designate this element of $\mathbb{S}_{n}$ by $\psi\left(i_{r}\right)$. Let $\Delta(i)=\sum_{r=0, \text { reven }}^{2 c} \psi\left(i_{r}\right)$, where the addition is coordinate-wise $(\bmod 2)$, so that $\Delta(i) \in \mathbb{S}_{n}$. Define $K\left({ }^{p} E_{i k}\right)=\phi^{-1}[\Delta(i)+\Delta(k)]$ to be the set $K_{\gamma}$ containing ${ }^{p} E_{i k}$.

Lemma 5.2. Suppose $p \in \Gamma,{ }^{p} E_{i h} \in K_{\alpha}$ and ${ }^{p} E_{h k} \in K_{\beta}$. Then ${ }^{p} E_{i k} \in K_{\alpha}$ $\otimes K_{\beta}=K_{\beta} \otimes K_{\alpha}$. Also, $K\left({ }^{p} E_{i k}\right)=K\left({ }^{p} E_{k i}\right)$.

Proof. The proof is immediate from the definitions of $\$ 5.1$ and the properties of the group $\left(S_{n}\right.$.

Lemma 5.3. Suppose $p \in \Gamma,{ }^{p} E_{i k} \in K_{\gamma}$. Let $i=i^{\prime} 2^{2 n}+i_{1} 2^{n}+i_{0}, k=k^{\prime} 2^{2 n}$ $+k_{1} 2^{n}+k_{0}\left(\right.$ with $\left.0 \leqq i_{1}, i_{0}, k_{1}, k_{0} \leqq 2^{n}-1\right)$, and write

$$
{ }^{p} E_{i k}={ }^{p} E\left(\left(i^{\prime}, i_{1}, i_{0}\right)\left(k^{\prime}, k_{1}, k_{0}\right)\right) \text {. }
$$

If ${ }^{p-2 n} E_{i^{\prime} k^{\prime}} \in K_{\alpha}$ and ${ }^{n} E_{i_{0} k_{0}} \in K_{\beta}$, then $K_{\gamma}=K_{\alpha} \otimes K_{\beta}$. Also,

$$
{ }^{p-2 n} E_{i^{\prime} i^{\prime}}^{p} E_{i k}^{p-2 n} R_{k^{\prime} k^{\prime}}={ }^{p} E_{i k} \text {. }
$$

Proof. If $p \in \Gamma$, then $p=(2 c+1) n$ for some fixed $c$. We show first that $\Delta(i)=\Delta\left(i^{\prime}\right)+\Delta\left(i_{0}\right)$. If $i^{\prime}=\sum_{r=0}^{2 c-2} i_{r}^{\prime} 2^{r n}$, then $\Delta\left(i^{\prime}\right)=\sum_{r=0, r \text { even }}^{2 c-2} \psi\left(i_{r}^{\prime}\right)$. Since 
$i=i^{\prime} 2^{2 n}+i_{1} 2^{n}+i_{0}$, it follows that $\Delta(i)=\sum_{r=0, r \text { even }}^{2 c-2} \psi\left(i_{r}^{\prime}\right)+\psi\left(i_{0}\right)=\Delta\left(i^{\prime}\right)$ $+\Delta\left(i_{0}\right)$. A similar argument shows that $\Delta(k)=\Delta\left(k^{\prime}\right)+\Delta\left(k_{0}\right)$. Hence

$$
\begin{aligned}
\phi\left(K_{\gamma}\right) & =\Delta(i)+\Delta(k)=\Delta\left(i^{\prime}\right)+\Delta\left(k^{\prime}\right)+\Delta\left(i_{0}\right)+\Delta\left(k_{0}\right) \\
& =\phi\left(K_{\alpha}\right)+\phi\left(K_{\beta}\right)=\phi\left(K_{\alpha} \otimes K_{\beta}\right),
\end{aligned}
$$

so $K_{\gamma}=K_{\alpha} \otimes K_{\beta}$.

Since $0 \leqq i_{1} 2^{n}+i_{0} \leqq 2^{2 n}-1$, we have $i=2^{2 n} i^{\prime}+a$, with $0 \leqq a \leqq 2^{2 n}-1$. Thus ${ }^{p} E_{i i} \leqq{ }^{p-2 n} E_{i^{\prime} i^{\prime}}$. Similarly, ${ }^{p} E_{k k} \leqq{ }^{p-2 n} E_{k^{\prime} k^{\prime}}$, and therefore the second statement holds.

Definition 5.4. Define the following classes of sets: $\mathscr{C}_{0}=\mathscr{N}_{0}=\left\{K_{0}\right\}$. For $j=1,2, \cdots, n, \mathscr{C}_{j}=\left\{K_{\gamma}: \gamma \equiv 0\left(\bmod 2^{n-j}\right)\right\}$ and $\mathscr{N}_{j}=\mathscr{C}_{j} \sim \mathscr{C}_{j-1}$.

Lemma 5.5. Suppose $j \geqq 1, p \in \Gamma$ and ${ }^{p} E_{r s} \in K_{h} \in \mathscr{N}_{h}$, with $0 \leqq h \leqq j$. Let ${ }^{p+2 n} E_{i k} \in K_{\gamma}$ be of the form ${ }^{p+2 n} E\left(\left(r, i_{1}, i_{0}\right)\left(s, k_{1}, k_{0}\right)\right)$. If $h \leqq j-1$, then $K_{\gamma} \in \mathscr{N}_{j}$ if and only if $i_{0} \equiv k_{0}\left(\bmod 2^{n-j}\right), i_{0} \not \equiv k_{0}\left(\bmod 2^{n-j+1}\right)$. If $h=j$, then $K_{\gamma} \in \mathscr{N}_{j}$ if and only if $i_{0} \equiv k_{0}\left(\bmod 2^{n-j+1}\right)$.

Proof. Let $K_{\beta}=K\left({ }^{n} E_{i_{0} k_{0}}\right)$. By Lemma $5.3, K_{\gamma}=K_{k} \quad K_{\beta}$. If $0 \leqq h \leqq$ $j-1$, then $\phi\left(K_{\gamma}\right)=\left(\cdots, C_{j-1}, 1,0, \cdots, 0\right)=\phi\left(K_{k}\right)+\phi\left(K_{\beta}\right)$ if and only if $\phi\left(K_{\beta}\right)=\psi\left(i_{0}\right)+\psi\left(k_{0}\right)=\left(\cdots, a_{j-1}, 1,0, \cdots, 0\right), \quad$ or $\quad i_{0} \equiv k_{0}\left(\bmod 2^{n-J}\right), i_{0} \not \equiv k_{0}$ $\left(\bmod 2^{n-J+1}\right)$.

If $h=j$, then $\phi\left(K_{\gamma}\right)=\left(\cdots, c_{j-1}, 1,0, \cdots, 0\right)$ if and only if $\phi\left(K_{\beta}\right)=$ $\left(\cdots, a_{j-1}, 0, \cdots, 0\right)$, or $i_{0} \equiv k_{0}\left(\bmod 2^{n-j+1}\right)$.

Definition 5.6. Let $\mathbf{R},\left\{U_{t}: t=1,2,3, \ldots\right\}$ be constructed as in $\S 2.2$, and let $K_{0}$ be defined as in $\$ 5.1$. If ${ }^{p} E_{i k} \in K_{0}(p \in \Gamma)$ implies $U_{p+h}^{p} E_{i k} U_{p+h}$ $={ }^{p} E_{i k}$ for all $h \geqq 1$, then $\mathbf{R}$ has property $\Lambda$.

Lemma 5.7. Suppose that $\mathbf{R}$ has property $\Lambda$. Let $V$ be a unitary in $\mathfrak{M}_{p}$ $(p \in \Gamma)$ such that $V^{[p]}=\sum \pm{ }^{p} E_{r s}$, where ${ }^{p} E_{r s}$ is in $K_{0}$ and the signs are arbitrary. Then $V$ leaves $\mathbf{R}$ invariant.

Proof. It is sufficient to show that $V$ leaves $\mathbf{S}$ invariant. Because of the structure of $\mathbf{S}$, we need only consider one of its generators, say ${ }^{p+t} \widetilde{E}_{k k}$ (see §2.2). Then

$$
\begin{aligned}
V^{p+t} \widetilde{E}_{k k} V^{*} & =\left[Y_{p} V^{|p|} Y_{p}^{*}\right] Y_{p+t}{ }^{p+t} E_{k k} Y_{p+t}^{*}\left[Y_{p} V^{|p|^{*}} Y_{p}^{*}\right] \\
& =Y_{p}\left[\sum \pm{ }^{p} E_{r s}\right] U_{p+1} \cdots U_{p+t}{ }^{p+t} E_{k k} U_{p+t} \cdots U_{p+1}\left[\sum \pm{ }^{p} E_{s r}\right] Y_{p}^{*} .
\end{aligned}
$$

By Lemma 5.2, ${ }^{p} E_{r s} \in K_{0}$ implies ${ }^{p} E_{s r} \in K_{0}$. So $U_{p+h}{ }^{p} E_{r s}={ }^{p} E_{r s} U_{p+h}$ and $U_{p+h}{ }^{p} E_{s r}={ }^{p} E_{s r} U_{p+h}$ for $h=1,2, \cdots, t$, by property $\Lambda$. Hence

$$
\left.V^{p+t} \widetilde{E}_{k k} V^{*}=Y_{p+t}\left[\sum \pm{ }^{p} E_{r s}\right]\right]^{p+t} E_{k k}\left[\sum \pm{ }^{p} E_{s r}\right] Y_{p+t}^{*}= \pm Y_{p+t}\left[{ }^{p+t} E_{r 0 r 0}\right] Y_{p+t}^{*}
$$

where $r_{0}$ depends on $k$. But by definition, this is also in $\mathbf{S}$. Therefore $V$ leaves $\mathbf{R}$ invariant.

Note. Since $K\left({ }^{p} E_{a b}\right)$ is independent of $p(p \in \Gamma)$, we use $K(a, b)$ as notation for $K\left({ }^{p} E_{a b}\right)$. 
Theorem 5.8. Suppose that $\mathbf{R}$ has property $\Lambda$. Let $\mathbf{P}=\mathfrak{N}(\mathbf{R})$ be as in $\S 1$. Then $\mathbf{P}$ is a factor.

Proof. Suppose $E \in \mathrm{P}, E$ commutes with everything in $\mathrm{P}, E \neq 0$ or $\alpha I$. Since $\mathbf{R} \subset \mathbf{P}$ and $\mathbf{R}$ is maximal abelian, we must have $E \in \mathbf{R}$. Since $E$ commutes with $E^{*}$ and hence is normal, it has a spectral resolution, and its spectral projections also commute with $\mathbf{P}$. So without loss of generality, we can suppose $E$ itself is a projection. If $0<[[E]]^{2} \leqq 1 / 2$, let $F=E$. If $1 / 2<[[E]]^{2}<1$, let $F=I-E$. Then $0<[[F]]^{2} \leqq 1 / 2$, and $F$ also commutes with $\mathbf{P}, F \in \mathbf{R}$.

By $[6$, p. 761$]$, there exists a sequence of projections $F_{m}$ which converges to $F$ in the metric topology, with $F_{m} \in \mathbf{S}$. Since $F \neq 0$, from some point on $F_{m} \neq 0$. Let $[[F]]^{2}=\delta$, and take $\epsilon=\delta / 2^{6}$. Choose $F_{m}$ such that $\left[\left[F_{m}-F\right]\right]$ $<\epsilon / 2$. If $V$ is any unitary in $\mathbf{P}$, then

$$
\left[\left[V F_{m}-F_{m} V\right]\right]=\left[\left[V F_{m}-V F+F V-F_{m} V\right]\right]<\epsilon .
$$

Also, since $0<\left[\left[F_{m}\right]\right],[[F]] \leqq 1$ and $\left|\left[\left[F_{m}\right]\right]-[[F]]\right|<\epsilon$, a straightforward calculation shows that $\left|\left[\left[F_{m}\right]\right]^{2}-[[F]]^{2}\right|<4 \epsilon=\delta / 2^{4}$. Hence $\delta-\delta / 2^{4}<\left[\left[F_{m}\right]\right]^{2}<\delta+\delta / 2^{4}$.

Without loss of generality, we may assume $\delta-\delta / 2^{4}<\left[\left[F_{m}\right]\right]^{2} \leqq 1 / 2$. (An analogous proof holds in the other case.) If $F_{m} \in \mathfrak{M}_{p}(p \in \Gamma)$, then $F_{m}^{[p]}=\sum_{H}{ }^{p} E_{r}$ for some subset $H \subset\left\{0,1, \cdots, 2^{p}-1\right\}$. It follows from the estimate of $\left[\left[F_{m}\right]\right]^{2}$ that $2^{p}\left(\delta-\delta / 2^{4}\right)<\#(H) \leqq 2^{p-1}$. For each $r \in H$, we can then choose $s(r), s=0,1, \cdots$, or $2^{p}-1, s \notin H$, such that if $r \neq r^{\prime}$, then $s(r) \neq s\left(r^{\prime}\right)$. Let $J=\{(r, s): r \in H, s=s(r)\}$, so that

$$
\#(J)=\#(H)>2^{p}\left(\delta-\delta / 2^{4}\right) \text {. }
$$

Consider ${ }^{p} E_{r s}$ for $(r, s) \in J$. Suppose $K(r, s)=K_{\alpha}$. If

$$
{ }^{p+2 n} E_{i k}={ }^{p+2 n} E\left(\left(r, i_{1}, i_{0}\right)\left(s, k_{1}, k_{0}\right)\right),
$$

then ${ }^{p+2 n} E_{i k} \in K_{\alpha} \otimes K\left(i_{0}, k_{0}\right)$, by Lemma 5.3. Hence ${ }^{p+2 n} E_{i k} \in K_{0}$ if and only if $\phi\left[K_{\alpha}\right]+\phi\left[K\left(i_{0}, k_{0}\right)\right]=\phi\left(K_{0}\right)$, or $\phi\left(K_{\alpha}\right)=\psi\left(i_{0}\right)+\psi\left(k_{0}\right)$. This equality clearly holds for exactly $2^{n}$ pairs $\left(i_{0}, k_{0}\right)$, where each index $0,1, \cdots, 2^{n}-1$ appears only once as first component and once as second. Let

$$
O(r, s)=\left\{\left(i_{0}, k_{0}\right): \phi\left(K_{\alpha}\right)=\psi\left(i_{0}\right)+\psi\left(k_{0}\right)\right\}
$$

and let $Z(r, s)=\left\{(i, k):{ }^{p+2 n} E_{i k}={ }^{p+2 n} E\left(\left(r, i_{1}, i_{0}\right)\left(s, k_{1}, k_{0}\right)\right), \quad i_{1}=k_{1}=0,1\right.$, $\cdots, 2^{n}-1$, and $\left.\left(i_{0}, k_{0}\right) \in O(r, s)\right\}$. Then $Z(r, s)$ contains $2^{2 n}$ pairs $(i, k)$, and for each of these, ${ }^{p+2 n} E_{i k}$ is in $K_{0}$. The sum $\sum_{(i, k) \in Z(r, s)}{ }^{p+2 n} E_{i k}$ contains not more than one nonzero element per row or column, since $i_{1}=k_{1}$. It follows, by definition of $J$, that $\sum_{(r, s) \in J}\left[\sum_{(i, k) \in Z(r, s)}{ }^{p+2 n} E_{i k}+{ }^{p+2 n} E_{k i}\right]$ also contains at most one nonzero element per row or column.

Therefore we can define a unitary $V$ such that

$$
V^{|p+2 n|}=\sum_{(r, s) \in J}\left[\sum_{(i, k) \in Z(r, s)}{ }^{p+2 n} E_{i k}+{ }^{p+2 n} E_{k i}\right]+\sum^{p+2 n} E_{t t},
$$


where the final sum is over all $t \neq i$ or $k$ if $(i, k)$ is in $Z(r, s)$ for any $(r, s)$ in $J$. All terms of $V^{[p+2 n]}$ are in $K_{0}$ so by Lemma 5.7, $V$ leaves $\mathbf{R}$ invariant, or $V \in \mathscr{V}$.

Then we have:

$$
\begin{aligned}
\epsilon^{2} & >\left[\left[V^{[p]} F_{m}^{[p]}-F_{m}^{[p]} V^{[p]}\right]\right]^{2} \quad(\text { by }(1)) \\
& =\left[\left[V^{[p]}\left(\sum_{H}^{p} E_{r r}\right)-\left(\sum_{H}^{p} E_{r r}\right) V^{[p]}\right]\right]^{2} \\
& =\left[\left[\sum_{r \in H, s \notin H}\left({ }^{p} E_{s s} V^{[p] p} E_{r r}-{ }^{p} E_{r r} V^{[p] p} E_{s s}\right)\right]\right]^{2} \\
& \geqq \sum_{(r, s) \in J}\left[\left[{ }^{p} E_{r r} V^{[p] p} E_{s s}\right]\right]^{2}=\sum_{(r, s) \in J}\left[\left[{ }^{p} E_{r r} V^{[p+2 n] p} E_{s s}\right]\right]^{2} \\
& =\sum_{(r, s) \in J}\left[\left[\sum_{(i, k) \in Z(r, s)}{ }^{p+2 n} E_{i k}\right]\right]^{2} \quad \text { (using Lemma 5.3) } \\
& =\sum_{J} 2^{2 n} / 2^{p+2 n}>\delta-\delta / 2^{4} \quad(\text { by }(2)) .
\end{aligned}
$$

This yields: $\delta / 2^{6}=\epsilon>\epsilon^{2}>\delta-\delta / 2^{4}>\delta / 2$, which is a contradiction. Hence the initial assumption that $0<[[F]]^{2} \leqq 1 / 2$ is incorrect, and we have either $[[E]]^{2}=0$ or $[[E]]^{2}=1$. Therefore $E=0$ or $I$, and so $\mathbf{P}$ is a factor.

Definition 5.9. Let $R\left(\mathscr{C}_{j}\right)(j=0,1, \cdots, n)$ be the ring generated by $\mathscr{X}_{j}=\left\{F: F \in \mathbb{M}_{p}(p \in \Gamma)\right.$ and $F^{[p]}={ }^{p} E_{a b}$ with $\left.K(a, b) \in \mathscr{C}_{j}\right\}$.

Theorem 5.10. Suppose that $\mathbf{R}$ has property $\Lambda$, and $\mathbf{P}$ and $\mathscr{V}$ are as in $\$ 1$. Suppose also that if $V \in \mathscr{V}$, then there is a sequence $V_{m} \in \mathfrak{M}$ converging metrically to $V$ such that if $V_{m} \in \mathfrak{M}_{p}(p \in \Gamma), \quad V_{m}^{[p]}=\sum \alpha_{a b}{ }^{p} E_{a b}$ with ${ }^{p} E_{a b}$ $\in K_{0}$. Then $\mathbf{P}=R\left(\mathscr{C}_{0}\right)$.

Proof. The collection of all unitaries $V$ which have the form required in Lemma 5.7 is sufficient to generate $\mathscr{X}_{0}$, hence $R\left(\mathscr{C}_{0}\right)$. Therefore $R\left(\mathscr{C}_{0}\right) \subset \mathbf{P}$. On the other hand, if $V \in \mathscr{V}$, then $V$ is in the metric closure of the subalgebra generated by the set $\mathscr{X}_{0}$, and hence $V$ is in its weak closure $R\left(\mathscr{C}_{0}\right)$. Thus $\mathbf{P} \subset R\left(\mathscr{C}_{0}\right)$, and so $\mathbf{P}=R\left(\mathscr{C}_{0}\right)$.

Definition 5.11. Let $\mathbf{R},\left\{U_{t}: t=1,2, \cdots\right\}$ be constructed as in $\S 2.2$. Suppose $p, q \in \Gamma, q>p$, and $A \in \mathfrak{M}_{p}$. If $A^{[p]}=\sum \alpha_{a b}{ }^{p} E_{a b}$ with $K(a, b)$ $\in \mathscr{N}_{j}$ implies $A^{[q]}=\sum \beta_{c d}{ }^{q} E_{c d}$ with $K(c, d) \in \mathscr{N}_{j}$, then $\mathbf{R}$ has property $\Omega$.

Lemma 5.12. Suppose $\mathbf{R}$ has property $\Omega$. If $F \in \mathbb{M}_{p}(p \in \Gamma), F^{[p]}={ }^{p} E_{a b}$ with $K(a, b) \in \mathscr{N}_{k}$, where $0 \leqq j<k$, then $F \underset{\leftarrow}{\leftarrow}\left(\mathscr{C}_{j}\right)$.

Proof. Suppose $F \in R\left(\mathscr{C}_{j}\right)$. Then there is a sequence $F_{m}$ converging strongly to $F$ such that if $F_{m} \in \mathfrak{M}_{q}(q \in \Gamma)$, then $F_{m}^{[q]}=\sum \delta_{c d}{ }^{q} E_{c d}$ with $K(c, d) \in \mathscr{C}_{j}$. Choose $F_{m}$ such that $\left[\left[F_{m}-F\right]\right]<1 / 2^{p}$, and choose $q$ so that $q \in \Gamma$ and $F_{m}, F \in \mathbb{M}_{q}$. By property $\Omega, F^{[q]}=\sum \alpha_{a b}{ }^{q} E_{a b}$ with $K(a, b) \in \mathscr{N}_{k}$. 
Since $k>j, \quad\left(F_{m}^{|q|}, F^{\mid q)}\right)=\left(F_{m}, F\right)=0$. So we have: $1 / 2^{2 p}>\left[\left[F_{m}-F\right]\right]^{2}$ $=\left[\left[F_{m}\right]\right]^{2}+[[F]]^{2}>1 / 2^{p}$, which is a contradiction. Therefore $F$ $\notin R\left(\mathscr{b}_{j}\right)$.

CoRollaRY 5.13. If $\mathbf{R}$ has property $\Omega$, then $R\left(\mathscr{C}_{j}\right) \varsubsetneqq R\left(\mathscr{C}_{j+1}\right)$ for any $j=0,1, \cdots, n-1$.

Proof. Clearly $\mathscr{X}_{j} \subset \mathscr{X}_{j+1}$, so $R\left(\mathscr{C}_{j}\right) \subset R\left(\mathscr{C}_{j+1}\right)$. But by Lemma 5.12 , $R\left(\mathscr{C}_{j}\right) \neq R\left(\mathscr{C}_{j+1}\right)$.

Definition 5.14. We construct $\mathbf{R}_{n}$ as in $\S 2.2$, with $\left\{U_{t}: t=1,2, \cdots\right\}$ defined as follows $\left(B_{t}\right.$ is as in \$4.1):

$U_{t}=I$ for $t \leqq n$. The rest of the unitaries are defined with reference to $p=(2 c+1) n$, or $p \in \Gamma$. If the matrix unit ${ }^{p} E_{0, r}$ is in $K_{\beta}$, then:

$$
\begin{aligned}
& { }^{p} E_{r r} U_{p+1}= \begin{cases}{ }^{p} E_{r} & \text { if } \beta \equiv 0(\bmod 2), \\
{ }^{p} E_{r} B_{p+1} & \text { if } \beta \equiv 1(\bmod 2) .\end{cases} \\
& { }^{p} E_{r} U_{p+n-k}= \begin{cases}{ }^{p} E_{r} & \text { if } \beta \equiv 0,1, \cdots, 2^{n-k-1}-1\left(\bmod 2^{n-k}\right), \\
{ }^{p} E_{r} B_{p+n-k} & \text { if } \beta \equiv 2^{n-k-1}, \cdots, 2^{n-k}-1\left(\bmod 2^{n-k}\right) .\end{cases} \\
& { }^{p} E_{r r} U_{p+n}= \begin{cases}{ }^{p} E_{r r} & \text { if } \beta \equiv 0,1, \cdots, 2^{n-1}-1\left(\bmod 2^{n}\right), \\
{ }^{p} E_{r} B_{p+n} & \text { if } \beta \equiv 2^{n-1}, \cdots, 2^{n}-1\left(\bmod 2^{n}\right) .\end{cases}
\end{aligned}
$$

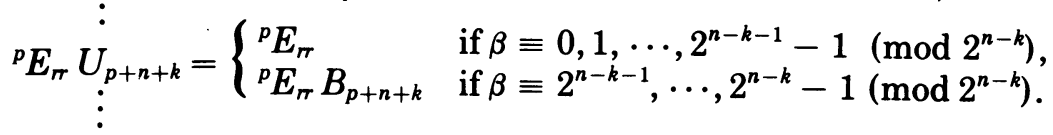

$$
\begin{aligned}
& U_{p+2 n}=I \text {. }
\end{aligned}
$$

Lemma 5.15. If $\left\{U_{l}: l=1,2, \cdots\right\}$ is constructed as in Definition 5.14, then $\mathbf{R}_{n}$ has property $\Lambda$.

Proof. Suppose $p \in \Gamma,{ }^{p} E_{i k} \in K_{0}$. If ${ }^{p} E_{0, i} \in K_{\beta}$ and ${ }^{p} E_{0, k} \in K_{\gamma}$, then $K_{\beta} \otimes K_{0}=K_{\gamma}$ implies $\beta=\gamma$.

(i) Suppose $h=1,2, \cdots, 2 n$. Then it is evident from Definition 5.14 that $U_{p+h}^{p} E_{i k} U_{p+h}=U_{p+h}^{p} E_{i i}^{p} E_{i k}^{p} E_{k k} U_{p+h}={ }^{p} E_{i k}$.

(ii) Suppose $h=2 t n+1,2 t n+2, \cdots, 2 t n+2 n$ for any positive integer $t$. Now ${ }^{p} E_{i k}=\sum^{p+2 t n} E_{i(t)+a, k(t)+a}$, where $i(t)=2^{2 n} i, k(t)=2^{2 n} k$, and the sum is over $a=0,1, \cdots, 2^{2 t n}-1$. A straightforward calculation based on $\$ 5.1$ and the properties of $\left(S_{n}\right.$ shows that each term of this sum is in $K_{0}$. If we let $p^{\prime}=p+2 t n \in \Gamma, h^{\prime}=1,2, \cdots, 2 n$, then we can apply part (i) to this situation, and conclude that $U_{p+h}{ }^{p} E_{i k} U_{p+h}={ }^{p} E_{i k}$ for all $h \geqq 1$.

Note. For the rest of this section, we assume $p \in \Gamma$.

Lemma 5.16. Suppose ${ }^{p} E_{r s} \in K_{\gamma}$ and $\phi\left(K_{\gamma}\right)=\left(a_{1}, a_{2}, \cdots, a_{n}\right)$. If $a_{k+1}=1$, then $U_{p+n \pm k}{ }^{p} E_{r s} U_{p+n \pm k}={ }^{p} E_{r s} B_{p+n \pm k}$. If $a_{k+1}=0$, then $U_{p+n \pm k}{ }^{p} E_{r s} U_{p+n \pm k}$ $={ }^{p} E_{r s}$.

Proof. Let $K_{\alpha}=K(0, r)$ and $K_{\beta}=K(0, s)$, so that $K_{\alpha} \otimes K_{\gamma}=K_{\beta}$. Suppose $a_{k+1}=1$. Then the $(k+1)$ st coordinate of $\phi\left(K_{\alpha}\right)$ does not equal the $(k+1)$ st 
coordinate of $\phi\left(K_{\beta}\right)$. So $\alpha \equiv y \in\left\{0,1, \cdots, 2^{n-k-1}-1\right\}\left(\bmod 2^{n-k}\right)$ if and only if $\beta \equiv z \in\left\{2^{n-k-1}, \cdots, 2^{n-k}-1\right\}\left(\bmod 2^{n-k}\right)$. Then Definition 5.14 shows that $U_{p+n \pm k}^{p} E_{r s} U_{p+n \pm k}={ }^{p} E_{r s} B_{p+n \pm k}$.

Suppose $a_{k+1}=0$. Then the $(k+1)$ st coordinate of $\phi\left(K_{\alpha}\right)$ equals the $(k+1)$ st coordinate of $\phi\left(K_{\beta}\right)$. So $\alpha \equiv y \in\left\{0,1, \cdots, 2^{n-k-1}-1\right\}\left(\bmod 2^{n-k}\right)$ if and only if $\beta \equiv z \in\left\{0,1, \cdots, 2^{n-k-1}-1\right\} \quad\left(\bmod 2^{n-k}\right)$. Thus in this case, $U_{p+n \pm k}^{p} E_{r s} U_{p+n \pm k}={ }^{p} E_{r s}$.

Lemma 5.17. Suppose ${ }^{p} E_{r s} \in K_{\gamma}$ and $\phi\left(K_{\gamma}\right)=\left(a_{1}, a_{2}, \cdots, a_{n}\right)$. Let $S$ $=\left\{k: a_{k+1}=1, k \neq 0\right\}$. If $a_{1}=1$, then

$$
U_{p+2 n} \ldots{ }^{p} E_{r s} \ldots U_{p+2 n}={ }^{p} E_{r s}\left[\prod_{k \in S} B_{p+n+k}\right] B_{p+n}\left[\prod_{k \in S} B_{p+n-k}\right] .
$$

If $a_{1}=0$, then

$$
U_{p+2 n} \ldots{ }^{p} E_{r s} \cdots U_{p+2 n}={ }^{p} E_{r s}\left[\prod_{k \in S} B_{p+n+k}\right]\left[\prod_{k \in S} B_{p+n-k}\right] .
$$

(Note. The operators $B_{p+t}$ commute.)

Proof. Using the fact that $B_{p+t}$ commutes with $U_{p+t+l}$ for $l \geqq 1$, we can apply Lemma 5.16 over and over again to perform the required calculation and obtain the results stated in this lemma.

Lemma 5.18. Suppose ${ }^{p} E_{r s} \in K_{\gamma}$ and $\phi\left(K_{\gamma}\right)=\left(a_{1}, a_{2}, \cdots, a_{n}\right)$. Define $\omega(\gamma)=2\left(\sum_{i=2}^{n} a_{i}\right)+a_{1}$. Then the nonzero entries of $U_{p+2 n} \ldots{ }^{p} E_{r s} \ldots U_{p+2 n}$ have numerical value $\pm(1 / \sqrt{ } 2)^{\omega(\gamma)}$.

Proof. This follows by direct calculation with the results of Lemma 5.17, because of the form of $B_{p+t}$.

LeMma 5.19. If $K\left({ }^{p} E_{r s}\right)=K_{\gamma} \in \mathscr{N}_{j}(j \geqq 1)$, then $U_{p+2 n} \cdots{ }^{p} E_{r s} \cdots U_{p+2 n}$ is in $\mathfrak{M}_{p+n+j-1}$. When written in $\mathfrak{M}_{p+2 n}$, it is made up of $2^{n-j+2}$ by $2^{n-j+2}$ blocks which are either zero or else of the form of Figure 1 , where $\beta=(1 / \sqrt{ } 2)^{\omega(\gamma)}$.

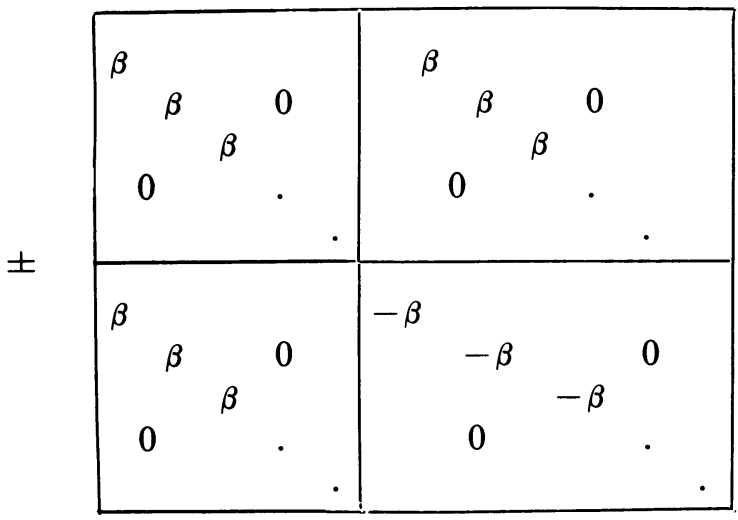

FIGURE 1 
Proof. Since $K_{\gamma} \in \mathscr{N}_{j}, \phi\left(K_{\gamma}\right)=\left(a_{1}, \cdots, a_{j-1}, 1,0, \cdots, 0\right)$. First take $j \neq 1$, so that $j-1 \neq 0$ and $j-1 \in S$. In fact, $j-1$ is the largest integer in $S$. So by Lemma $5.17, \quad U_{p+2 n} \cdots{ }^{p} E_{r s} \cdots U_{p+2 n}={ }^{p} E_{r s} B_{p+n+j-} \cdots B_{p+n-j+1}$, where the subscripts are in decreasing order. Hence the product is in $\mathfrak{M}_{p+n+j-1}$.

Now

$$
\begin{aligned}
{ }^{p} E_{r s} B_{p+n+j-1} \cdots B_{p+n-j+1} & =B_{p+n+j-1}\left[{ }^{p} E_{r s} \cdots B_{p+n-j+1}\right] \\
& =B_{p+n+j-1}\left[\sum \pm \alpha^{p+n+j-2} E_{a b}\right] \\
& =\sum \pm \alpha^{p+n+j-2} E_{a b} B_{p+n+j-1}
\end{aligned}
$$

(for certain pairs $(a, b)$ ). This is made up of 2 by 2 blocks which are either zero or else of the form $\pm \alpha B_{1}$. Hence, when written in $\mathfrak{M}_{p+2 n}$, the product is made up of $2^{n-j+2}$ by $2^{n-j+2}$ blocks which are either zero or else have the the form indicated in the lemma. Here $\beta=\alpha / \sqrt{ } 2=(1 / \sqrt{ } 2)^{\omega(\gamma)}$, by Lemma 5.18 .

If $j=1$, then $U_{p+2 n} \cdots{ }^{p} E_{r s} \cdots U_{p+2 n}={ }^{p} E_{r s} B_{p+n}$, and the preceding paragraph applies to this case also.

Lemma 5.20. Let $\left\{U_{t}: t=1,2, \cdots\right\}$ be constructed as in Definition 5.14. Then $\mathbf{R}_{n}$ has property $\Omega$ (see Definition 5.11 ).

Proof. Let $A \in \mathfrak{M}_{p}, q>p, p$ and $q$ in $\Gamma . A^{[q]}=U_{q} \ldots U_{p+1} A^{[p]} U_{p+1} \cdots U_{q}$, and because of linearity it is sufficient to consider $U_{q} \ldots{ }^{p} E_{r s} \ldots U_{q}$, with $K(r, s) \in \mathscr{N}_{j}$.

Suppose $j=0$. Since $\mathbf{R}_{n}$ has property $\Lambda$ (Lemma 5.15), $U_{q} \ldots{ }^{p} E_{r s} \cdots U_{q}$ $={ }^{p} E_{r s}$. But ${ }^{p} E_{r s}=\sum_{i}{ }^{q} E_{r_{i} s_{i}}$, where $r_{i}=2^{q-p} r+i, \quad s_{i}=2^{q-p} s+i$, and $i$ $=0,1, \cdots, 2^{q-p}-1$. Again, a calculation based on $\$ 5.1$ shows that each term of this sum is in $K_{0}$.

Suppose $j \geqq 1$. Then Lemma 5.19 asserts that $U_{p+2 n} \cdots{ }^{p} E_{r s} \ldots U_{p+2 n}$ $=\sum \beta_{c d}^{p+2 n} E_{c d}$ is in $\mathfrak{M}_{p+n+j-1}$. Consider any term of this sum. If we write ${ }^{p+2 n} E_{c d} \operatorname{as}^{p+2 n} E\left(\left(r, i_{1}, i_{0}\right)\left(s, k_{1}, k_{0}\right)\right)$, then $\left(i_{0}, k_{0}\right)=\left(2^{n-(j-1)} i^{\prime \prime}+a, 2^{n-(j-1)} k^{\prime \prime}+a\right)$ for some $i^{\prime \prime}, k^{\prime \prime}, a\left(0 \leqq a \leqq 2^{n-J+1}-1\right)$. Hence $i_{0} \equiv k_{0} \quad\left(\bmod 2^{n-j+1}\right)$. But $K(r, s) \in \mathscr{N}_{j}$, so by Lemma $5.5, K(c, d) \in \mathscr{N}_{j}$. An extension by induction shows that $R_{n}$ has property $\Omega$.

Lemma 5.21. Suppose $\mathbf{R}_{n}$ is constructed as in Definition 5.14, and $\mathscr{V}$ $=\left\{V: V \mathbf{R}_{n} V^{*}=\mathbf{R}_{n}, V\right.$ unitary, $\left.V \in \mathfrak{A}\right\}$. If $V \in \mathscr{V}$, then there is a sequence $V_{m} \in \mathbb{M}$ converging metrically to $V$, such that if $V_{m} \in \mathbb{M}_{p}(p \in \Gamma), \quad V_{m}^{[p]}$ $=\sum \alpha_{c d}^{p} E_{c d}$ with ${ }^{p} E_{c d} \in K_{0}$.

Proof. By Lemma 4.3, it is sufficient to show that $\mathbf{R}_{n}$ satisfies the conditions of $\$ 4.1$.

Suppose $p \in \Gamma$ and consider ${ }^{p} E_{a b}$. We showed in Lemma 5.15 that if 
${ }^{p} E_{a b} \in K_{0}$, then $U_{p+h}{ }^{p} E_{a b} U_{p+h}={ }^{p} E_{a b}$ for $h \geqq 1$. If ${ }^{p} E_{a b} \notin K_{0}$, then $K(a, b)$ $\in \mathscr{N}_{j}$ for some $j \geqq 1$, so that $\phi[K(a, b)]=\left(\cdots, c_{j-1}, 1,0, \cdots, 0\right)$. Therefore $U_{p+n-j+1}^{p} E_{a b} U_{p+n-j+1}={ }^{p} E_{a b} B_{p+n-j+1}$, by Lemma 5.16 . If we let $d(a, b)$ $=n-j+1$, then $1 \leqq j \leqq n$ implies $1 \leqq d \leqq n$. Lemma 5.16 also asserts that $U_{p+n-k}{ }^{p} E_{a b} U_{p+n-k}={ }^{p} E_{a b}$ for $k=j, j+1, \cdots, n-1$, or $n-k \leqq d$. Hence the conditions in the second paragraph of $\$ 4.1$ are satisfied.

We recall that $\mathscr{N}_{0}=\left\{K_{0}\right\}$, and that if $K_{\gamma} \in \mathscr{N}_{j}$ for $j \geqq 1$, then $K_{\gamma} \neq K_{0}$. Thus Lemma 5.20 is an explicit statement of the final condition of $\$ 4.1$.

Theorem 5.22. Let $\mathbf{R}_{n}$ be constructed as in Definition 5.14. Then $\mathbf{R}_{n}$ is semi-regular but not regular.

Proof. Let $\mathbf{P}_{n}=\mathfrak{N}\left(\mathbf{R}_{n}\right)$. Since $\mathbf{R}_{n}$ has property $\Lambda$ (Lemma 5.15), Theorem 5.8 shows that $P_{n}$ is a factor. Because of Lemma 5.21, Theorem 5.10 applies to $\mathbf{R}_{n}$, and $\mathbf{P}_{n}=R\left(\mathscr{C}_{0}\right)$. But $n \geqq 1$ and $R\left(\mathscr{C}_{0}\right) \varsubsetneqq R\left(\mathscr{C}_{1}\right) \subset \mathscr{A}$ (by Corollary 5.13), so $\mathbf{P}_{n}=R\left(\mathscr{C}_{0}\right) \neq \mathscr{A}$. Therefore $\mathbf{R}_{n}$ is semi-regular but not regular.

We now proceed to show that if $\theta$ is a ${ }^{*}$-automorphism of $\mathfrak{A}$ and $n_{1} \neq n_{2}$, then we cannot have $\theta\left(\mathbf{R}_{n_{1}}\right)=\mathbf{R}_{n_{2}}$. For the rest of this section we assume $\left\{U_{t}: t=1,2, \cdots\right\}$ constructed as in Definition 5.14.

Lemma 5.23. Suppose ${ }^{p} E_{r s} \in K_{\alpha}$ and ${ }^{p} E_{s t} \in K_{\beta}$, with $K_{\alpha}$ and $K_{\beta}$ in $\mathscr{N}_{k}$ for some $k \geqq 1$. If $\phi\left(K_{\alpha}\right)=\left(a_{1}, \cdots, a_{n}\right)$ and $\phi\left(K_{\theta}\right)=\left(b_{1}, \cdots, b_{n}\right)$, define $\mu(\alpha, \beta)$ $=2\left(\sum_{i=2}^{k-1} a_{i} b_{i}\right)+a_{1} b_{1}+a_{k} b_{k}$. Let $r_{0}=2^{n+k-2} r, s_{0}=2^{n+k-2} s, t_{0}=2^{n+k-2} t$. Then $2^{\mu}$ is the number of distinct $\delta$ 's such that

$$
{ }^{p+n+k-2} E_{r_{0} r_{0}}\left[U_{p+2 n} \ldots{ }^{p} E_{r s} \cdots U_{p+2 n}\right]^{p+n+k-2} E_{s_{0}+\delta, s_{0}+\delta}
$$

and

$$
{ }^{p+n+k-2} E_{s_{0} \delta_{0}}\left[U_{p+2 n} \cdots{ }^{p} E_{r s} \cdots U_{p+2 n}\right]^{p+n+k-2} E_{t_{0}+\delta, t_{0}+\delta}
$$

are both nonzero.

Proof. If we consider the results of Lemma 5.17, then $\mu$ is the number of factors $B_{p+h}(1 \leqq h \leqq p+n+k-2)$ which $U_{p+n+k-2} \ldots{ }^{p} E_{r s} \ldots U_{p+n+k-2}$ and $U_{p+n+k-2} \ldots{ }^{p} E_{s t} \ldots U_{p+n+k-2}$ have in common. If the first of these products equals $\sum \rho_{a b}{ }^{p+n+k-2} E_{a b}$ and the second equals $\sum \eta_{c d}{ }^{p+n+k-2} E_{c d}$, then $2^{\mu}$ is the number of $\delta$ 's such that the first sum contains the pair $(a, b)$ $=\left(r_{0}, s_{0}+\delta\right)$ while the second contains the pair $(c, d)=\left(s_{0}, t_{0}+\delta\right)$. (This is a result of straightforward calculation with the operators $B_{p+h}$.) Since $K_{\omega}, K_{\beta} \in \mathscr{N}_{k}$, we have $a_{k}=b_{k}=1$ and $a_{k+1}, \cdots, a_{n}, b_{k+1}, \cdots, b_{n}=0$. So by Lemma 5.16 (and the commuting properties of $B_{p+n+k-1}$ ),

$$
U_{p+2 n} \ldots{ }^{p} E_{r s} \cdots U_{p+2 n}=\sum \rho_{a b}{ }^{p+n+k-2} E_{a b} B_{p+n+k-1}
$$

and

$$
U_{p+2 n} \cdots{ }^{p} E_{s t} \cdots U_{p+2 n}=\sum \eta_{c t}{ }^{p+n+k-2} E_{c t} B_{p+n+k-1} .
$$


Thus $2^{\mu}$ is also the number of $\delta^{\prime}$ s such that the two expressions in question in the lemma are both nonzero.

Lemma 5.24. Suppose ${ }^{p} E_{r s} \in K_{\alpha},{ }^{p} E_{s t} \in K_{\beta}$, and $K_{\alpha}, K_{\beta} \in \mathscr{N}_{k}(k \geqq 1)$. Let $\omega_{1}=\omega(\alpha), \omega_{2}=\omega(\beta)$, and $\mu=\mu(\alpha, \beta)$. (See Lemmas 5.18 and 5.23.) If $K_{\gamma}=K_{\alpha} \otimes K_{\beta}$, then the function $C_{k}(\gamma)=2^{\mu}(1 / \sqrt{ } 2)^{\omega_{1}+\omega_{2}}$ depends only on $k$ and on $K_{\gamma}$.

Proof. If $\phi\left(K_{\alpha}\right)=\left(a_{1}, \cdots, a_{n}\right)$ and $\phi\left(K_{\beta}\right)=\left(b_{1}, \cdots, b_{n}\right)$, then $\phi\left(K_{\alpha} \otimes K_{\beta}\right)$ $=\phi\left(K_{\gamma}\right)=\left(a_{1}+b_{1}, a_{2}+b_{2}, \cdots, a_{n}+b_{n}\right)(\bmod 2)$. Suppose $K_{\alpha^{\prime}} \otimes K_{\beta^{\prime}}=K_{\gamma}$, with $K_{\alpha^{\prime}}, K_{\beta^{\prime}} \in \mathscr{N}_{k}$. If $\phi\left(K_{\alpha^{\prime}}\right)=\left(a_{1}^{\prime}, \cdots, a_{n}^{\prime}\right)$ and $\phi\left(K_{\beta^{\prime}}\right)=\left(b_{1}^{\prime}, \cdots, b_{n}^{\prime}\right)$, then $a_{i}^{\prime}+b_{i}^{\prime} \equiv a_{i}+b_{i}(\bmod 2)$ for $i=1,2, \cdots, n$.

Let $\left(\omega_{1}+\omega_{2}\right)_{i}$ be the contribution to $\omega_{1}+\omega_{2}$ from $a_{i}$ and $b_{i}$, and let $\mu_{i}$ be the contribution to $\mu$ from $a_{i}$ and $b_{i}$, so that $\omega_{1}+\omega_{2}=\sum_{i=1}^{k}\left(\omega_{1}+\omega_{2}\right)_{i}$ and $\mu=\sum_{i=1}^{k} \mu_{i}$.

Suppose $a_{i}+b_{i}=a_{i}^{\prime}+b_{i}^{\prime}=1$. Then $a_{i} \neq b_{i}$ and $a_{i}^{\prime} \neq b_{i}^{\prime}$, so that $\mu_{i}=\mu_{i}^{\prime}$ and $\left(\omega_{1}+\omega_{2}\right)_{i}=\left(\omega_{1}^{\prime}+\omega_{2}^{\prime}\right)_{i}$.

Suppose $a_{i}+b_{i} \equiv a_{i}^{\prime}+b_{i}^{\prime} \equiv 0$. If $a_{i}, b_{i}=a_{i}^{\prime}, b_{i}^{\prime}$, then clearly $\mu_{i}=\mu_{i}^{\prime}$ and $\left(\omega_{1}+\omega_{2}\right)_{i}=\left(\omega_{1}^{\prime}+\omega_{2}^{\prime}\right)_{i}$. If $a_{i}, b_{i}=1$ and $a_{i}^{\prime}, b_{i}^{\prime}=0$, with $i=1,2, \cdots$, or $k-1$, then direct computation shows that

$$
2^{\mu_{i}}(1 / \sqrt{ } 2)^{\left(\omega_{1}+\omega_{2}\right)_{i}}=2^{\mu_{i}^{\prime}}(1 / \sqrt{ } 2)^{\left(\omega_{1}+\omega_{2}\right)_{i}}=1 \text {. }
$$

(We need not consider $i=k$, since $a_{k}, b_{k}=a_{k}^{\prime}, b_{k}^{\prime}=1$.) The same computation and result apply if $a_{i}, b_{i}=0$ and $a_{i}^{\prime}, b_{i}^{\prime}=1$.

Therefore

$$
\begin{aligned}
2^{\mu}(1 / \sqrt{ } 2)^{\left(\omega_{1}+\omega_{2}\right)} & =\prod_{i} 2^{\mu_{i}}(1 / \sqrt{ } 2)^{\left(\omega_{1}+\omega_{2}\right)_{i}} \\
& =\prod_{i} 2^{\mu_{i}^{\prime}}(1 / \sqrt{ } 2)^{\left(\omega_{1}+\omega_{2}\right)_{i}} \quad \text { (since individual factors are equal) } \\
& =2^{\mu_{i}^{\prime}}(1 / \sqrt{ } 2)^{\left(\omega_{1}+\omega_{2}\right)} .
\end{aligned}
$$

So this function depends only on $k$ and on $K_{\gamma}$.

Lemma 5.25. For each $k=2,3, \cdots, n$, let $P_{k}(s)$ be the operator such that $P_{k}(s)^{[p+2 n]}=\sum_{h}{ }^{p+2 n} E_{s^{\prime \prime}+h, s^{\prime \prime}+h}$, where $s^{\prime \prime}=2^{2 n} s, h \equiv 0\left(\bmod 2^{n-k+2}\right)$, and $0 \leqq h$ $\leqq 2^{2 n}-1$. Suppose ${ }^{p} E_{r s} \in K_{\alpha},{ }^{p} E_{s t} \in K_{\beta}, K_{\alpha}$ and $K_{\beta}$ in $\mathscr{N}_{k}$, and $K_{\alpha} \otimes K_{\beta}$ $=K_{\gamma}$. Then

$$
\left[U_{p+2 n} \cdots{ }^{p} E_{r s} \cdots U_{p+2 n}\right] P_{k}(s)^{\lfloor p+2 n]}\left[U_{p+2 n} \ldots{ }^{p} E_{s t} \cdots U_{p+2 n}\right]
$$

has a term of the form $C_{k}(\gamma)^{p+2 n} E_{r^{n}, t^{n+}+\pi}$ and a term of the form $C_{k}(\gamma)^{p+2 n} E_{r^{n}+\pi, t^{n+\pi}}$, where $r^{\prime \prime}=2^{2 n} r, t^{\prime \prime}=2^{2 n} t$, and $\pi=2^{n-k+1}$. If $K_{\gamma} \in \mathscr{C}_{k-2}$, then $K\left(r^{\prime \prime}, t^{\prime \prime}+\pi\right)$ $\in \mathscr{N}_{k-1}$. If $K_{\gamma} \in \mathscr{N}_{k-1}$, then $K\left(r^{\prime \prime}+\pi, t^{\prime \prime}+\pi\right) \in \mathscr{N}_{k-1}$.

Proof. We use the notation of Lemma 5.23. Let $\delta_{1}$ be a typical index which has the property that 


$$
{ }^{p+n+k-2} E_{r_{0} r_{0}}\left[U_{p+2 n} \cdots{ }^{p} E_{r s} \cdots U_{p+2 n}\right]^{p+n+k-2} E_{s_{0}+\delta_{1}, s_{0}+\delta_{1}}
$$

is nonzero, and $\delta_{2}$ one with the property that

$$
{ }^{p+n+k-2} E_{s_{0} s_{0}}\left[U_{p+2 n} \cdots{ }^{p} E_{s t} \cdots U_{p+2 n}\right]^{p+n+k-2} E_{t_{0}+\delta_{2}, t_{0}+\delta_{2}}
$$

is nonzero. Since $U_{p+1}, \cdots, U_{p+2 n}$ are all self-adjoint,

$$
{ }^{p+n+k-2} E_{s_{0}+\delta_{2}, s_{0}+\delta_{2}}\left[U_{p+2 n} \cdots{ }^{p} E_{s t} \ldots U_{p+2 n}\right]^{p+n+k-2} E_{t_{0} t_{0}}
$$

is also nonzero. For $i=1$ or 2 , let $h\left(\delta_{i}\right)=2^{n-k+2} \delta_{i} \equiv 0\left(\bmod 2^{n-k+2}\right)$. Finally, let $\omega_{1}=\omega(\alpha), \omega_{2}=\omega(\beta)$, and $\mu=\mu(\alpha, \beta)$. Then

$$
\begin{aligned}
{ }^{p+2 n} E_{r^{\prime \prime}, r^{\prime \prime}}\left[\left(^{*}\right)\right]^{p+2 n} E_{t^{\prime \prime}+\pi, t^{\prime \prime}+\pi} & \left.=\left[\sum_{h\left(\delta_{1}\right)}(1 / \sqrt{ } 2)^{\omega_{1}\left({ }^{p+2 n}\right.} E_{r^{\prime \prime}, s^{\prime \prime}+h\left(\delta_{1}\right)}\right)\right]\left[\sum_{h\left(\delta_{2}\right)}(1 / \sqrt{ } 2)^{\left.\omega_{2}\left(p^{p+2 n} E_{s^{\prime \prime}+h\left(\delta_{2}\right), t^{*}+\pi}\right)\right] .}\right.
\end{aligned}
$$

These terms are the only nonzero ones after applying the projections, and the signs are all positive by an obvious calculation. In the product of these two sums, there are nonzero terms if and only if $h\left(\delta_{1}\right)=h\left(\delta_{2}\right)$. So by Lemma 5.23 , there are $2^{\mu}$ nonzero terms. Hence the entire product equals $2^{\mu}(1 / \sqrt{ } 2)^{\omega_{1}+\omega_{2}}\left({ }^{p+2 n} E_{r^{\prime \prime}, t^{\prime \prime}+\pi}\right)$, where the scalar is equal to $C_{k}(\gamma)$, by Lemma 5.24.

A similar argument gives the other term.

If $K_{\gamma} \in \mathscr{C}_{k-2}$, then $K_{\gamma} \in \mathscr{N}_{i}$ for some $i \leqq k-2$. Now

$$
{ }^{p+2 n} E_{r^{n}, t^{n}+\pi}={ }^{p+2 n} E\left((r, 0,0)\left(t, 0,2^{n-k+1}\right)\right) \text {. }
$$

So by Lemma $5.5, K\left(r^{\prime \prime}, t^{\prime \prime}+\pi\right) \in \mathscr{N}_{k-1}$. If $K_{\gamma} \in \mathscr{N}_{k-1}$, then ${ }^{p+2 n} E_{r^{\prime \prime}+\pi, t^{\prime \prime}+\pi}$ $={ }^{p+2 n} E\left(\left(r, 0,2^{n-k+1}\right)\left(t, 0,2^{n-k+1}\right)\right)$ implies that $K\left(r^{\prime \prime}+\pi, t^{\prime \prime}+\pi\right) \in \mathscr{N}_{k-1}$, again by Lemma 5.5 .

LeMmA 5.26. Suppose $k \geqq 2,{ }^{p} E_{r s} \in K_{\alpha} \in \mathscr{N}_{k-1},{ }^{p} E_{s t} \in K_{\beta} \in \mathscr{C}_{k-2}$ (or vice versa). Then, using the notation of Lemma 5.25,

$$
{ }^{p+2 n} E_{r^{n+\pi}, r^{*+}+\pi}\left[\left(^{*}\right)\right]^{p+2 n} E_{t^{n+\pi}+t^{n+\pi}}=0 .
$$

Proof. By Lemma 5.19, $U_{p+2 n} \ldots{ }^{p} E_{r s} \ldots U_{p+2 n}$ is in $\mathfrak{M}_{p+n+k-2}$. So it has no elements of the form ${ }^{p+2 n} E_{r^{n}+x, s^{n}+h}, h \equiv 0\left(\bmod 2^{n-k+2}\right)$. Since $P_{k}(s)^{[p+2 n]}$ $=\sum^{p+2 n} E_{8^{n}+h, s^{\prime \prime}+h}, h \equiv 0\left(\bmod 2^{n-k+2}\right)$, the product in question in this lemma must be zero. (A similar proof holds if the roles of $K_{\alpha}$ and $K_{\beta}$ are exchanged.)

Lemma 5.27. Suppose $k \geqq 2$ and $W \in \mathfrak{M}_{p}$ is such that $W^{[p]}=V^{[p]}+X^{[p]}$, with $V^{[p]}=\sum \beta_{r s}^{p} E_{r s}\left(K(r, s) \in \mathscr{C}_{k-1}\right)$ and $X^{[p]}=\sum \alpha_{r s}{ }^{p} E_{r s}\left(K(r, s) \in \mathscr{N}_{k}\right)$. Let $(r, t)$ be a fixed pair such that ${ }^{p} E_{r t} \in K_{\gamma} \in \mathscr{C}_{k-1}$. Then the product

$$
\begin{aligned}
{ }^{p} E_{\pi} & {\left[U_{p+2 n} \cdots W^{[p]} \cdots U_{p+2 n}\right] \sum_{s=0}^{2^{p}-1} P_{k}(s)^{[p+2 n]}\left[U_{p+2 n} \cdots W^{*[p]} \cdots U_{p+2 n}\right]^{p} E_{t t} } \\
= & A(r, t)^{[p+2 n]}+Q(r, t)^{[p+2 n]}
\end{aligned}
$$


where $\left(A^{[p+2 n]}, \quad Q^{[p+2 n)}\right)=0$, and $Q^{[p+2 n]}=\sum_{s=0}^{2^{p}-1} \alpha_{r s} \bar{\alpha}_{t s} C_{k}(\gamma)^{p+2 n} E_{a b}$ with $K(a, b) \in \mathscr{N}_{k-1}$.

Proof. The product $\left({ }^{* *}\right)$ equals

$$
\sum_{s=0}^{2^{p}-1}\left[U_{p+2 n} \cdots \delta_{r s}^{p} E_{r s} \cdots U_{p+2 n}\right] P_{k}(s)^{[p+2 n]}\left[U_{p+2 n} \cdots \bar{\delta}_{t s}^{p} E_{s t} \cdots U_{p+2 n}\right],
$$

since $P_{k}(s)^{\lfloor p+2 n\rfloor}={ }^{p} E_{s s} P_{k}(s)^{\lfloor p+2 n] p} E_{s s}$ and ${ }^{p} E_{r r},{ }^{p} E_{s s},{ }^{p} E_{t t}$ commute with $U_{p+l}(l \geqq 1)$.

Case 1. $K_{\gamma} \in \mathscr{C}_{k-2}$. Suppose $s$ is such that $K(r, s)$ and $K(s, t)$ are both in $\mathscr{N}_{k}$. The summand corresponding to this $s$ includes the term $\alpha_{r s} \bar{\alpha}_{t s} C_{\mathrm{k}}(\gamma)$ $X^{p+2 n} E_{r^{n}, t^{\prime \prime}+\pi}$, according to Lemma 5.25, which also asserts that this matrix unit is contained in a set of $\mathscr{N}_{k-1}$. Consider the summands corresponding to other $s$. The only possible way for them to yield something not orthogonal to a matrix unit in $\mathscr{N}_{k-1}$ would be to have $K(r, s) \in \mathscr{N}_{k-1}$ and $K(s, t)$ $\in \mathscr{C}_{k-2}$ (or vice versa), since $P_{k}(s)^{[p+2 n]}$ is a sum of matrix units in $K_{0}$. But then $K(r, t) \in \mathscr{N}_{k-1}$, contradicting $K(r, t)=K_{\gamma} \in \mathscr{C}_{k-2}$.

Hence $\sum_{s=0}^{2^{p}-1} \alpha_{r s} \bar{\alpha}_{t s} C_{k}(\gamma)^{p+2 n} E_{r^{\prime \prime}, t^{\prime \prime}+\pi}$ gives the desired $Q(r, t)^{[p+2 n]}$.

Case 2. $K_{\gamma} \in \mathscr{N}_{k-1}$. Again suppose $s$ is such that $K(r, s), K(s, t) \in \mathscr{N}_{k}$. The summand for this $s$ includes the term $\alpha_{r s} \bar{\alpha}_{t s} C_{k}(\gamma)^{p+2 n} E_{r^{n+\pi}, t^{*+}}$, according to Lemma 5.25, and again this matrix unit is in a set of $\mathscr{N}_{k-1}$. So summands for other $s$ 's can yield something not orthogonal to it only if $K(r, s)$ $\in \mathscr{N}_{k-1}$ and $K(s, t) \in \mathscr{C}_{k-2}$ (or vice versa). But Lemma 5.26 assures us that these summands cannot have entries in row $r^{\prime \prime}+\pi$, column $t^{\prime \prime}+\pi$.

Hence $\sum_{s=0}^{2^{p}-1} \alpha_{r s} \bar{\alpha}_{t s} C_{k}(\gamma)^{p+2 n} E_{r^{\prime \prime}+\pi, t^{\prime \prime}+\pi}$ gives the desired $Q(r, t)^{[p+2 n]}$.

Corollary 5.28. Suppose $k \geqq 2, W$ and $(r, t)$ as in Lemma 5.27, and $q>p(p, q \in \Gamma)$. Then

$$
{ }^{p} E_{r r} W^{[q]} \sum_{s=0}^{2 p-1} P_{k}(s)^{[q]} W^{*[q] p} E_{t}=A(r, t)^{[q]}+Q(r, t)^{[q]},
$$

where $\left(A^{[q]}, Q^{[q]}\right)=0, Q^{[q]}=\sum \rho_{c d}{ }^{q} E_{c d}$ with $K(c, d) \in \mathscr{N}_{k-1}$, and $[[Q]]^{2}$ $\geqq\left|\sum_{s=0}^{2 p-1} \alpha_{r s} \bar{\alpha}_{t s}\right|^{2} / 2^{p+6 n-4} \quad$ (where the constants are from the sum defining $X^{[p]}$.

Proof. We first obtain a lower bound for the function $C_{k}(\gamma)$. Clearly $\mu \geqq a_{k} b_{k}=1$ and $\omega_{1}+\omega_{2} \leqq 2(2 j-1)=4 j-2$. Hence $C_{k}(\gamma)=2^{\mu}(1 / \sqrt{ } 2)^{\omega_{1}+\omega_{2}}$ $\geqq(1 / 2)^{2 n-2}$.

By Lemma 5.27, the corollary holds if $q=p+2 n$, since

$$
[[Q]]^{2}=\left[\left[Q^{[p+2 n]}\right]\right]^{2}=\left|C_{k}(\gamma)\right|^{2}\left|\sum_{s=0}^{2 p-1} \alpha_{r s} \bar{\alpha}_{t s}\right|^{2} / 2^{p+2 n} \geqq\left|\sum_{s} \alpha_{r s} \bar{\alpha}_{t s}\right|^{2} / 2^{p+6 n-4}
$$

Now 


$$
\begin{aligned}
{ }^{p} E_{r r} & W^{[q]} \sum_{s} P_{k}(s)^{[q]} W^{*[q] p} E_{t t} \\
& =U_{q} \cdots U_{p+2 n+1}\left[{ }^{p} E_{r r} W^{[p+2 n]} \sum_{s} P_{k}(s)^{[p+2 n]} W^{*[p+2 n] p} E_{t t}\right] U_{p+2 n+1} \cdots U_{q} \\
& =U_{q} \cdots U_{p+2 n+1}\left[A(r, t)^{[p+2 n]}+Q(r, t)^{[p+2 n]}\right] U_{p+2 n+1} \cdots U_{q} \\
& =A(r, t)^{[q]}+Q(r, t)^{[q]} .
\end{aligned}
$$

The unitaries preserve the orthogonality of $A$ and $Q$ and the norm of $Q$. Also, by Lemma 5.20, $\quad Q^{[p+2 n]}=\rho^{p} E_{a b}$ with $K(a, b) \in \mathscr{N}_{k-1}$ implies $Q^{[q]}$ $=\sum \rho_{c d}^{q} E_{c d}$ with $K(c, d) \in \mathscr{N}_{k-1}$.

Lemma 5.29. Suppose $W \in \mathfrak{M}_{p}$ is such that $W^{[p]}=V^{[p]}+X^{[p]}$, with $V^{[p]}=\sum \beta_{r s}^{p} E_{r s}\left(K(r, s) \in \mathscr{C}_{k-1}\right)$ and $X^{[p]}=\sum \alpha_{r s}^{p} E_{r s}\left(K(r, s) \in \mathscr{N}_{k}\right)$. Then $\|V\| \leqq\|W\|$ and $\|X\| \leqq\|W\|+\|V\|$.

Proof. If $K(r, s) \in \mathscr{C}_{k-1}$, then $\phi[K(0, r)]=\left(a_{1}, \cdots, a_{k-1}, a_{k}, \cdots, a_{n}\right)$ and $\phi[K(0, s)]=\left(b_{1}, \cdots, b_{k-1}, a_{k}, \cdots, a_{n}\right)$. So both of these $n$-tuples are of the form $\left(\cdots, a_{k}, \cdots, a_{n}\right)$, where the first $k-1$ coordinates are arbitrary and the last $n-k+1$ are fixed. Call the set of all such $n$-tuples $\kappa$. Define the projection $E_{\kappa}=\sum{ }^{p} E_{u u}$, where the sum is over all $u$ such that $\phi[K(0, u)]$ is in $\kappa$. Then ${ }^{p} E_{r s}=E_{\kappa}{ }^{p} E_{r s}={ }^{p} E_{r s} E_{\kappa}=E_{\kappa}{ }^{p} E_{r s} E_{\kappa}$.

In general: Let $\kappa_{i}$ be the set of all $n$-tuples $\left(\cdots, a_{k}^{(i)}, \cdots, a_{n}^{(i)}\right)$, where the last $n-k+1$ coordinates are fixed for each $i$. If we sum over the projections $E_{\kappa_{i}}$ for all possible sets $\kappa_{i}$, then $V^{[p]}=\sum_{i} E_{\kappa_{i}} W^{[p]} E_{\kappa_{i}}$. If $\kappa_{1} \neq \kappa_{2}$, then $E_{\kappa_{1}}$ is orthogonal to $E_{\kappa_{2}}$, since $K(0, u)$ is uniquely determined. Hence $\left\|V^{[p]}\right\|=\sup _{i}\left\|E_{\kappa_{i}} W^{[p]} E_{\kappa_{i}}\right\| \leqq\left\|W^{[p]}\right\|$, and $\|V\| \leqq\|W\|$.

Since $X=W-V,\|X\| \leqq\|W\|+\|V\|$.

Lemma 5.30. For $j=1,2, \cdots, n$, let $R\left(\mathscr{C}_{j-1}\right)$ be as in Definition 5.9, and let $\mathscr{V}_{j}=\left\{V: V\left[R\left(\mathscr{C}_{j-1}\right)\right] V^{*}=R\left(\mathscr{C}_{j-1}\right), \quad V\right.$ unitary, $\left.V \in \mathfrak{A}\right\} . \quad$ If $V \in \mathscr{V}_{j}$, then there is a sequence $V_{m} \in \mathbb{M}$ converging metrically to $V$ such that if $V_{m}$ $\in \mathbb{M}_{p}, \quad V_{m}^{\{p\rfloor}=\sum \beta_{r s}^{p} E_{r s}$ with $K(r, s) \in \mathscr{C}_{j}$.

Proof. (i) Since $V$ is in $\mathfrak{A},\|V\| \leqq 1$, there is a sequence $W_{m}$ in $\mathfrak{M},\left\|W_{m}\right\|$ $\leqq 1$, which converges strongly to $V[3]$. Strong convergence implies metric convergence, so $\lim _{m}\left[\left[W_{m}-V\right]\right]=0$. If $W_{m} \in \mathfrak{M}_{p}$, then $W_{m}^{[p]}$ clearly equals $\sum \beta_{r s}{ }^{p} E_{r s}$ with $K(r, s) \in \mathscr{C}_{n}$.

(ii) To show: Suppose $j<k \leqq n$, and suppose there exists $W_{m} \in \mathfrak{M}$ such that $\left\|W_{m}\right\| \leqq 1, \lim _{m}\left[\left[W_{m}-V\right]\right]=0$, and $W_{m} \in \mathfrak{M}_{p}$ implies $W_{m}^{\{p]}$ $=\sum \delta_{r s}{ }^{p} E_{r s}$ with $K(r, s) \in \mathscr{C}_{k}$. Then there exists $V_{m}$ with the same properties except that $V_{m}^{\lfloor p \mid}=\sum \beta_{r s}^{p} E_{r s}$ with $K(r, s) \in \mathscr{C}_{k-1}$.

We let the assumed $W_{m}^{[p]}=V_{m}^{[p]}+X_{m}^{[p]}$, where

$$
V_{m}^{[p]}=\sum \beta_{r s}{ }^{p} E_{r s} \quad\left(K(r, s) \in \mathscr{C}_{k-1}\right) \quad \text { and } X_{m}^{[p]}=\sum \alpha_{r s}^{p} E_{r s} \quad\left(K(r, s) \in \mathscr{N}_{k}\right) .
$$

Since $\left(V_{m}, X_{m}\right)=0, X_{m}$ is Cauchy in the metric topology. Also, $\left\|W_{m}\right\| \leqq 1$ 
implies $\left\|V_{m}\right\| \leqq 1$ and $\left\|X_{m}\right\| \leqq 2$, by Lemma 5.29. A slight adaptation of a proof of Murray and von Neumann [6, p. 723] then shows that $X_{m}$ is also Cauchy in the strong topology and has a strong limit $X \in \mathfrak{A}$. Since $X^{*}$ $\in \mathscr{U}$ also, $X$ and $X^{*}$ bounded operators, it is easy to verify that

$$
\left[\left[X_{m} X_{m}^{*}-X X^{*}\right]\right] \rightarrow 0 \text {. }
$$

Suppose $\lim _{m}\left[\left[X_{m}\right]\right] \neq 0$. Then $[[X]] \neq 0, X \neq 0, X X^{*} \neq 0$, and so $\lim _{m}\left[\left[X_{m} X_{m}^{*}\right]\right] \neq 0$. Hence $\left[\left[X_{m} X_{m}^{*}\right]\right]^{2}>2^{6 n} \epsilon^{2}$ for all $m$ and some $\epsilon>0$.

Choose $W_{m}$ such that $\left[\left[W_{m}-V\right]\right]<\epsilon / 4$. Suppose $W_{m} \in \mathfrak{M}_{p}$. Then $X_{m}^{[p]}=\sum \alpha_{r s}{ }^{p} E_{r s}\left(K(r, s) \in \mathscr{N}_{k}\right)$ implies

$$
\left(1 / 2^{p}\right) \sum\left|\sum_{s=0}^{2 p_{-}} \alpha_{r s} \bar{\alpha}_{t s}\right|^{2}>2^{6 n} \epsilon^{2} .
$$

(The outer summation is over pairs $(r, t)$ such that $K(r, t) \in \mathscr{C}_{k-1}$, since $K(r, s), K(s, t) \in \mathscr{N}_{k}$.) Fix $p$ from here on.

For every $s=0,1, \ldots, 2^{p}-1$, define $P_{k}(s)$ as in Lemma 5.25. We drop the subscript $k$ for the rest of this proof and consider $\sum_{s=0}^{2^{p}} P(s)^{[p+2 n]}$. Since ${ }^{p+2 n} E_{s^{n}+h, s^{\prime \prime}+h}$ is in $K_{0}, \sum_{s} P(s)$ is in $R\left(\mathscr{C}_{j-1}\right)$ for any $j \geqq 1$. Hence if $V \in \mathscr{V}_{j}, V\left(\sum_{s} P(s)\right) V^{*}=T \in R\left(\mathscr{L}_{j-1}\right)$, where $T$ depends on $k$. So there exists a sequence $T_{\nu} \in \mathfrak{M}$, $\left[\left[T_{\nu}-T\right]\right] \rightarrow 0$, and $T_{\nu} \in \mathfrak{M}_{q}(q \in \Gamma)$ implies $T^{\lfloor q\rfloor}$ $=\sum \eta_{i h}{ }^{9} E_{i h}$ with $K(i, h) \in \mathscr{C}_{j-1}$. Choose $T_{\nu}$ such that $\left[\left[V\left(\sum_{8} P(s)\right) V^{*}-T_{\nu}\right]\right]$ $<\epsilon / 2$, and take $q$ such that $T_{\nu} \in \mathfrak{M}_{q}, q \geqq p+2 n, q \in \Gamma$. Then

$$
\left[\left[V^{[q]}\left(\sum_{s} P(s)^{[q]}\right) V^{*[q]}-T_{v}^{[q]}\right]\right]<\epsilon / 2 .
$$

Since $\sum_{s} P(s)^{[p+2 n]}$ is a projection, so is $\sum_{s} P(s)^{[q]}$, and hence $\left\|\sum_{s} P(s)^{[q]}\right\|$ $\leqq 1$. Thus

$$
\begin{aligned}
{\left[\left[W_{m}^{[q]}\right.\right.} & \left.\left.\sum P(s)^{[q]} W_{m}^{*[q]}-V^{[q]} \sum P(s)^{[q]} V^{*[q]}\right]\right] \\
& \leqq\left[\left[W_{m}^{[q]} \sum P(s)^{[q]}\left(W_{m}^{*[q]}-V^{*[q]}\right)\right]\right]+\left[\left[\left(W_{m}^{[q]}-V^{[q]}\right) \sum P(s)^{[q]} V^{*[q]}\right]\right] \\
& \leqq 2\left[\left[W_{m}^{*[q]}-V^{*[q]}\right] 1<\epsilon / 2 .\right.
\end{aligned}
$$

It follows from estimates (2) and (3) that

$$
\left[\left[W_{m}^{[q]} \sum P(s)^{[q]} W_{m}^{*[q]}-T_{\nu}^{[q]}\right]\right]<\epsilon .
$$

On the other hand, if $K(r, t) \in \mathscr{C}_{k-1}(k \geqq j+1 \geqq 2)$ and $q \in \Gamma, q>p$, then we can apply Corollary 5.28 , with $W_{m}$ replacing $W$. Since $Q^{|q|}$ $=\sum \rho_{\text {od }}^{q} E_{\text {cd }}\left(K(c, d) \in \mathcal{N}_{k-1}\right)$ and $T_{v}^{[q]}=\sum \eta_{i h}^{q} E_{i h}\left(K(i, h) \in \mathscr{C}_{j-1}\right)$, where $k-1>j-1$, we have $\left(T_{v}^{[q]}, Q^{[q]}\right)=0$ also. Therefore

$$
\begin{aligned}
{\left[\left[{ }^{p} E_{r r} W_{m}^{[q]} \sum\right.\right.} & \left.\left.P(s)^{[q]} W_{m}^{*[q] p} E_{t t}-{ }^{p} E_{r} T_{v}^{[q] p} E_{t t}\right]\right]^{2} \\
& =\left[\left[A(r, t)^{[q]}+Q(r, t)^{[q]}-{ }^{p} E_{r} T_{v}^{[q] p} E_{t t}\right]\right]^{2} \\
& \geqq\left[\left[Q(r, t)^{[q]}\right]\right]^{2} \geqq\left.\left|\sum_{s} \alpha_{r s} \bar{\alpha}_{t s}\right|\right|^{2} / 2^{p+6 n-4} .
\end{aligned}
$$


So we have, finally:

$$
\begin{aligned}
\epsilon^{2} & >\left[\left[W_{m}^{[q]} \sum P(s)^{[q]} W_{m}^{*[q]}-T_{v}^{[q]}\right]\right]^{2} \quad(\text { by }(4)) \\
& \geqq \sum_{(r, t)}\left[\left[{ }^{p} E_{r r}\left(W_{m}^{[q]} \sum P(s)^{[q]} W_{m}^{*[q]}-T_{v}^{[q]}\right)^{p} E_{t t}\right]\right]^{2}, \quad \text { where } K(r, t) \in \mathscr{C}_{k-1} \\
& \geqq \sum_{(r, t)}\left|\sum \alpha_{r s} \bar{\alpha}_{t s}\right|^{2} / 2^{p+6 n-4} \quad(\text { by }(5)) \\
& >16 \epsilon^{2} \quad(\text { by }(1)) .
\end{aligned}
$$

But this is a contradiction. Therefore $\lim _{l}\left[\left[X_{l}\right]\right]=0$. Since $\lim _{l}\left[\left[V_{l}-X_{l}-V\right]\right]$ $=0$, we have $\lim _{l}\left[\left[V_{l}-V\right]\right]=0$, where $\left\|V_{l}\right\| \leqq 1$, and $V_{l} \in \mathfrak{M}_{z}(z \in \Gamma)$ implies $V_{l}=\sum \beta_{r s}{ }^{2} E_{r s}$ with $K(r, s) \in \mathscr{C}_{k-1}$.

Since we can extend this as far as $k=j+1$ by a finite induction process, the lemma is proved.

THEOREM 5.31. If $j \geqq 1$, and $R(\mathscr{\mathscr { V }})$ is the ring generated by the set $\mathscr{V}_{j}$ of Lemma 5.30, then $R\left(\mathscr{V}_{j}\right)=R\left(\mathscr{C}_{j}\right)$.

Proof. To show: $R\left(\mathscr{C}_{j}\right) \subset R\left(\mathscr{V}_{j}\right)$. Let $T \in R\left(\mathscr{C}_{j-1}\right)$. Suppose $V_{1} \in \mathfrak{M}_{p}$, $V_{1}^{[p]}=\sum \pm{ }^{p} E_{r s}$, where $K(r, s) \in \mathscr{C}_{j-1}$ and the signs are arbitrary. Since $V_{1}$ and $V_{1}^{*}$ are then in $R\left(\mathscr{C}_{j-1}\right), V_{1} T V_{1}^{*} \in R\left(\mathscr{C}_{j-1}\right)$ and $V_{1} \in \mathscr{V}_{j}$.

Suppose $V_{2} \in \mathfrak{M}_{p}, V_{2}^{[p]} \sum \pm{ }^{p} E_{r s}$, where $K(r, s) \in \mathscr{N}_{j}$ and the signs are arbitrary. There exists a sequence $T_{m} \in \mathfrak{M}$ converging strongly to $T$ such that if $T_{m} \in \mathfrak{M}_{q}(q \in \Gamma)$, then

$$
T_{m}^{[q]}=\sum \beta_{c d}^{q} E_{c d}\left(K(c, d) \in \mathscr{C}_{j-1}\right) .
$$

If $z=\max [p, q]$, then

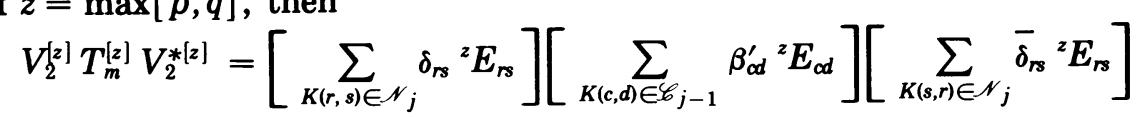

by Lemma 5.20. Now if $K_{j}, K_{j^{\prime}} \in \mathscr{N}_{j}$ and $K_{h} \in \mathscr{C}_{j-1}$, then $K_{j} \otimes K_{h} \otimes K_{j^{\prime}}$ $\in \mathscr{C}_{j-1}$, calculating by means of the isomorphism of $\S 5.1$. So each matrix unit of the product is in a set of $\mathscr{C}_{j-1}$, and $V_{2} T_{m} V_{2}^{*} \in R\left(\mathscr{C}_{j-1}\right)$. Since $V_{2}$ is fixed, $V_{2} T_{m} V_{2}^{*}$ converges strongly to $V_{2} T V_{2}^{*}$, which is then also in $R\left(\mathscr{C}_{j-1}\right)$. Therefore $V_{2} \in \mathscr{V}_{j}$.

But the collection of all unitaries which have the form of either $V_{1}$ or $V_{2}$ is sufficient to generate $R\left(\mathscr{C}_{j}\right)$ (see Definition 5.9 ), and so $R\left(\mathscr{C}_{j}\right)$ $\subset R\left(\mathscr{V}_{j}\right)$.

On the other hand, $R\left(\mathscr{V}_{j}\right) \subset R\left(\mathscr{C}_{j}\right)$ by Lemma 5.30 , and therefore $R\left(\mathscr{V}_{j}\right)$ $=R\left(\mathscr{C}_{j}\right)$.

Theorem 5.32. With $\mathbf{R}_{n}$ constructed as in Definition 5.14 , let $L=$ length of $\mathbf{R}_{n}$ be as in $\S 1$. Then $L\left(\mathbf{R}_{n}\right)=n$.

Proof. $\mathbf{P}_{n}$ is a factor, $\mathbf{R}_{n}$ is abelian and $\mathbf{R}_{n} \neq\{\alpha I\}$, and so $\mathbf{R}_{n} \varsubsetneqq \mathbf{P}_{n}$.

To show: $\mathfrak{N}^{j}\left(\mathbf{P}_{n}\right)=R\left(\mathscr{C}_{j}\right)$ for $j \geqq 0$. (i) By Lemmas 5.15 and 5.21 and 
Theorem 5.10, $\mathfrak{N}^{0}\left(\mathbf{P}_{n}\right)=\mathbf{P}_{n}=R\left(\mathscr{C}_{0}\right)$. (ii) Assume $\mathfrak{N}^{k-1}\left(\mathbf{P}_{n}\right)=R\left(\mathscr{C}_{k-1}\right)$, for $1 \leqq k \leqq j$. Then $\mathfrak{N}^{k}\left(\mathbf{P}_{n}\right)=\mathfrak{N}\left[\mathfrak{N}^{k-1}\left(\mathbf{P}_{n}\right)\right]=\mathfrak{N}\left[R\left(\mathscr{C}_{k-1}\right)\right]=R\left(\mathscr{C}_{k}\right)$, using Theorem 5.31. Therefore, by induction, $\mathfrak{N}^{j}\left(\mathbf{P}_{n}\right)=R\left(\mathscr{C}_{j}\right)$.

Since $R\left(\mathscr{C}_{j}\right) \varsubsetneqq R\left(\mathscr{C}_{j+1}\right)$ (Corollary 5.13$), \mathfrak{N}^{j}\left(\mathbf{P}_{n}\right) \varsubsetneqq \mathfrak{N}^{j+1}\left(\mathbf{P}_{n}\right)$ for $0 \leqq j$ $\leqq n-1$. Also, it is evident from the definitions that $\mathfrak{N}^{n}\left(\mathbf{P}_{n}\right)=R\left(\mathscr{C}_{n}\right)=\mathfrak{U}$. Therefore $L\left(\mathbf{R}_{n}\right)=n$.

Theorem 5.33. Let $\mathbf{R}_{n_{1}}$ and $\mathbf{R}_{n_{2}}$ be constructed as in Definition 5.14, with $n_{1} \neq n_{2}$. Then there cannot exist $a^{*}$-automorphism $\theta$ of $\mathfrak{A}$ such that $\theta\left(\mathbf{R}_{n_{1}}\right)$ $=\mathbf{R}_{n_{2}}$.

Proof. A standard argument shows that if $\mathbf{D}_{1}$ and $\mathbf{D}_{2}$ are subrings of $\mathfrak{U}$ and $\theta$ a ${ }^{*}$-automorphism of $\mathfrak{A}$ such that $\theta\left(\mathbf{D}_{1}\right)=\mathbf{D}_{2}$, then $\theta\left[\mathfrak{N}\left(\mathbf{D}_{1}\right)\right]$ $=\mathfrak{N}\left[\theta\left(\mathbf{D}_{1}\right)\right]=\mathfrak{N}\left(\mathbf{D}_{2}\right)$. It follows that $L$ is an invariant under *atomorphisms of $\mathfrak{A}$. But by Theorem 5.32, $L\left(\mathbf{R}_{n_{1}}\right)=n_{1}$ and $L\left(\mathbf{R}_{n_{2}}\right)=n_{2}$. So we cannot have $\theta\left(\mathbf{R}_{n_{1}}\right)=\mathbf{R}_{n_{2}}$.

In the section we have therefore constructed an infinite sequence of semi-regular (but not regular) maximal abelian subalgebras of $\mathfrak{A}$ which cannot be pairwise connected by ${ }^{*}$-automorphisms of $\mathfrak{A}$.

6. A counter example: another semi-regular subalgebra. We present here an example $\mathbf{R}^{\prime}$ which shows that the invariant $L$ does not give rise to a complete classification of semi-regular subalgebras. In the construction of $\mathbf{R}^{\prime}$, we assume the definitions of $\$ 5.1$ and Definition 5.4 with $n=2$, so that Lemmas 5.2, 5.3, and 5.5 hold.

Definition 6.1. We construct $\mathbf{R}^{\prime}$ as in $\$ 2.2$, with $\left\{U_{t}: t=1,2, \cdots\right\}$ defined as follows $\left(B_{t}\right.$ is as in $\left.\$ 4.1\right)$ :

$U_{t}=I$ for $t \leqq n=2$. The rest of the unitaries are defined with reference to $p=(2 c+1) n=4 c+2$, or $p \in \Gamma$. If the matrix unit ${ }^{p} E_{0, r}$ is in $K_{\beta}$, then:

$$
\begin{aligned}
{ }^{p} E_{r} U_{p+1} & = \begin{cases}{ }^{p} E_{r r} & \text { if } \beta \equiv 0(\bmod 2), \\
{ }^{p} E_{r r} B_{p+1} & \text { if } \beta \equiv 1(\bmod 2) .\end{cases} \\
{ }^{p} E_{r r} U_{p+2} & = \begin{cases}{ }^{p} E_{r r} & \text { if } \beta \equiv 0,1\left(\bmod 2^{2}\right), \\
{ }^{p} E_{r r} B_{p+2} & \text { if } \beta \equiv 2,3\left(\bmod 2^{2}\right) .\end{cases} \\
U_{p+3} & =U_{p+4}=I .
\end{aligned}
$$

Lemma 6.2. If $\left\{U_{t}: t=1,2, \cdots\right\}$ is constructed as in Definition 6.1, then $\mathbf{R}^{\prime}$ has property $\Lambda$ (Definition 5.6).

Proof. The proof is like that of Lemma 5.15.

Lemma 6.3. Let $\left\{U_{t}: t=1,2, \cdots\right\}$ be as in Definition 6.1, and suppose $p, q \in \Gamma, q>p$, and $T \in \mathbb{M}_{p}$. If $T^{[p]}=\sum \delta_{a b}{ }^{p} E_{a b}$ with $K(a, b)=K_{\gamma}$ $(\gamma=0,1,2$, or 3$)$, then $T^{|q|}=\sum \beta_{c d}{ }^{q} E_{c d}$ with $K(c, d)=K_{\gamma}$, and so $\mathbf{R}^{\prime}$ has property $\Omega$ (Definition 5.11). 
Proof. $T^{[q]}=U_{q} \ldots U_{p+1} T^{[p]} U_{p+1} \cdots U_{q}$, and by linearity it is sufficient to consider $U_{q} \ldots{ }^{p} E_{a b} \ldots U_{q}, K(a, b)=K_{r}$.

We show that the lemma holds for $q=p+4$, and the rest follows by an obvious induction argument. Now $U_{p+3}=U_{p+4}=I$, and so $U_{p+4} \cdots{ }^{p} E_{a b}$ $\ldots U_{p+4}=\sum \beta_{c d}{ }^{p+4} E_{o d}$ is in $\mathfrak{M}_{p+2}$. Consider one term. If we write ${ }^{p+4} E_{c d}$ $={ }^{p+4} E\left(\left(a, i_{1}, i_{0}\right)\left(b, i_{1}, i_{0}\right)\right)$, then $\left(i_{0}, k_{0}\right)=\left(2^{2} i^{\prime \prime}+v, 2^{2} j^{\prime \prime}+v\right)$ for some $i^{\prime \prime}$, $j^{\prime \prime}, v(0 \leqq v \leqq 3)$. Hence $i_{0} \equiv k_{0}\left(\bmod 2^{2}\right)$, or $i_{0}=k_{0}$, so $K\left(i_{0}, k_{0}\right)=K_{0}$. Therefore $K(c, d)=K(a, b) \otimes K\left(i_{0}, k_{0}\right)=K(a, b)=K_{r}$.

Note. The property of Lemma 6.3 is stronger than property $\Omega$ and will be used to show that $L\left(\mathbf{R}^{\prime}\right)=1 \neq L\left(\mathbf{R}_{2}\right)$.

Lemma 6.4. Suppose $\mathbf{R}^{\prime}$ is constructed as in Definition 6.1 and $\mathscr{V}=\left\{V: V \mathbf{R}^{\prime} V^{*}\right.$ $=\mathbf{R}^{\prime}, V$ unitary, $\left.V \in \mathfrak{A}\right\}$. If $V \in \mathscr{V}$, there is a sequence $V_{m} \in \mathfrak{M}$ converging metrically to $V$, such that if $V_{m} \in \mathfrak{M}_{p}(p \in \Gamma), \quad V_{m}^{[p]}=\sum \alpha_{a b}{ }^{p} E_{a b}$ with ${ }^{p} E_{a b}$ $\in K_{0}$.

Proof. By Lemma 4.3, it is sufficient to show that $\mathbf{R}^{\prime}$ satisfies the conditions of $\$ 4.1$.

Suppose $p \in \Gamma$ and consider ${ }^{p} E_{i k}$. Lemma 6.2 states that if ${ }^{p} E_{i k} \in K_{0}$, then $U_{p+h}{ }^{p} E_{i k} U_{p+h}={ }^{p} E_{i k}$ for $h \geqq 1$. Suppose ${ }^{p} E_{i k} \notin K_{0}$, but $K(i, k) \in \mathscr{N}_{j}$ for $j=1$ or 2. If $K_{\alpha}=K(0, i)$ and $K_{\beta}=K(0, k)$, then $\alpha \equiv \beta\left(\bmod 2^{2-}\right)$ while $\alpha \not \equiv \beta\left(\bmod 2^{2-j+1}\right)$, by using $\$ 5.1$. So Definition 6.1 shows that $U_{p+2-j+1}^{p} E_{i k} U_{p+2-j+1}={ }^{p} E_{i k} B_{p+2-j+1}$. If we take $d(i, k)=2-j+1$, then $1 \leqq d \leqq 2$. If $d=2$, then $j=1$ and $\alpha \equiv \beta(\bmod 2)$. So by Definition 6.1, $U_{p+1}^{p} E_{i k} U_{p+1}={ }^{p} E_{i k}$. Hence the conditions in the second paragraph of $\$ 4.1$ are satisfied.

Finally, Lemma 6.3 is an explicit statement of the final condition of $\$ 4.1$.

Lemma 6.5. Let $\mathbf{R}^{\prime}$ be constructed as in Definition 6.1. Then $\mathbf{R}^{\prime}$ is semiregular but not regular.

Proof. Theorems 5.8 and 5.10 and Corollary 5.13 apply here, too, so the proof is like that of Theorem 5.22 .

Theorem 6.6. $\mathbf{R}^{\prime} \varsubsetneqq \mathbf{P}^{\prime} \varsubsetneqq \mathfrak{N}\left(\mathbf{P}^{\prime}\right)=\mathfrak{A}$, and so $L\left(\mathbf{R}^{\prime}\right)=1$.

Proof. Since $\mathbf{P}^{\prime}$ is a factor and $\mathbf{R}^{\prime}$ is abelian, $\mathbf{R}^{\prime} \neq \mathbf{P}^{\prime}$. Also, by Theorem $6.5, \mathbf{P}^{\prime} \neq \mathfrak{A}$. So we need only show that $\mathfrak{N}\left(\mathbf{P}^{\prime}\right)=\mathfrak{A}$.

Let $T \in R\left(\mathscr{C}_{0}\right)=\mathbf{P}^{\prime}$. Then there is a sequence $T_{m}$ converging strongly to $T$, such that if $T_{m} \in \mathfrak{M}_{q}(q \in \Gamma)$, then $T_{m}^{[q]}=\sum \beta_{c d}{ }^{q} E_{c d}$ with $K(c, d)$ $=K_{0}$. Suppose $V_{\gamma} \in \mathfrak{M}_{p}(p \in \Gamma), V_{\gamma}^{[p]}=\sum \pm{ }^{p} E_{r s}$ with $K(r, s)=K_{\gamma}(\gamma=0$, $1,2$, or 3$)$ for all terms in the sum. If $z=\max [p, q]$, then

$$
V_{\gamma}^{[z]} T_{m}^{[z]} V_{\gamma}^{*[z]}=\left[\sum_{K(r, s)=K \gamma} \delta_{r s}{ }^{2} E_{r s}\right]\left[\sum_{K(c, d)=K_{0}} \beta_{c d}^{\prime}{ }^{2} E_{\alpha d}\right]\left[\sum_{K(s, r)=K_{\gamma}} \bar{\delta}_{r s}{ }^{z} E_{s r}\right]
$$


by Lemma 6.3. But $K_{\gamma} \otimes K_{0} \otimes \mathrm{K}_{\gamma}=K_{0}$, and so each matrix unit in this product is in $K_{0}$, and $V_{\gamma} T_{m} V_{\gamma}^{*} \in R\left(\mathscr{C}_{0}\right)$. Since $V_{\gamma} T_{m} V_{\gamma}^{*}$ converges strongly to $V_{\gamma} T V_{\gamma}^{*}$, this is also in $R\left(\mathscr{C}_{0}\right)$, and $V_{\gamma}$ leaves $\mathbf{P}^{\prime}$ invariant.

But the collection of all unitaries which have the form of $V_{\gamma}$ (for $\gamma=0$, 1,2 , and 3 ) is sufficient to generate $R\left(\mathscr{C}_{2}\right)$ (see Theorem 5.10$)$, and $R\left(\mathscr{C}_{2}\right)$ $=\mathfrak{A}$. Therefore $\mathfrak{A}=R\left(\mathscr{C}_{2}\right) \subset \mathfrak{N}\left(\mathbf{P}^{\prime}\right)$, and $\mathfrak{N}\left(\mathbf{P}^{\prime}\right)=\mathfrak{A}$.

Lemma 6.7. Let $\mathbf{R}_{1}$ be as in Definition 5.14, $\mathbf{R}^{\prime}$ as in Definition 6.1. Then there cannot exist $a^{*}$-automorphism $\theta$ of $\mathfrak{A}$ such that $\theta\left(\mathbf{R}^{\prime}\right)=\mathbf{R}_{1}$, although $L\left(\mathbf{R}^{\prime}\right)=L\left(\mathbf{R}_{1}\right)=1$.

Proof. We show first that there cannot exist a ${ }^{*}$-automorphism $\theta$ of $\mathfrak{A}$ such that $\theta\left(\mathbf{P}^{\prime}\right)=\mathbf{P}_{1}$. The invariant is the fact that the product of two operators which are in $\mathbf{P}_{1}^{\perp}$ is always in $\mathbf{P}_{1}$.

Using the definitions of $\$ 5.1$ and Definition 5.4 for $n=2$, let $S_{1}$ and $S_{2}$ be in $\mathfrak{M}_{p}(p \in \Gamma)$ with

$$
S_{1}^{[p]}={ }^{p} E_{a b}\left(K(a, b) \in \mathscr{N}_{1}\right) \quad \text { and } \quad S_{2}^{[p]}={ }^{p} E_{c d}\left(K(c, d) \in \mathscr{N}_{2}\right) .
$$

Then $S_{1}^{\lfloor p\rfloor} S_{2}^{\lfloor p\rfloor}=\left(S_{1} S_{2}\right)^{[p\rfloor}=\sum \delta_{w v}^{p} E_{w v}\left(K(u, v) \in \mathscr{N}_{2}\right)$. So $S_{1}, S_{2}$, and $S_{1} S_{2}=S_{3}$ are all in $R\left(\mathscr{C}_{0}\right)^{\perp}=\mathbf{P}^{\prime \perp}$.

Let $\theta$ be a ${ }^{*}$-automorphism of $\mathscr{A}$, and suppose $\theta\left(S_{i}\right)=T_{i}$ for $i=1,2$, or 3. Then $T_{i} \in \mathcal{U}$, so $\left\|T_{i}\right\|<\infty$. By [3], we can choose a sequence $T_{i_{v}}$ $\in \mathfrak{M}$ converging strongly to $T_{i}$ with $\left\|T_{i_{\nu}}\right\| \leqq\left\|T_{i}\right\|$. We now use $\$ 5.1$ and Definition 5.4 with $n=1$. If $T_{i_{v}} \in \mathbb{M}_{q}(q \in \Gamma)$, let $T_{i_{v}}^{[q]}=P_{i_{\nu}}^{[q]}+X_{i_{v}}^{[q]}$, where $P_{i_{v}}^{[q]}=\sum \rho_{j k}{ }^{q} E_{j k}\left(K(j, k) \in \mathscr{C}_{0}\right)$ and $X_{i_{\nu}}^{[q]}=\sum \eta_{r s}{ }^{q} E_{r s}\left(K(r, s) \in \mathscr{N}_{1}\right)$. Then $P_{i_{v}} \in \mathbf{P}_{1}$ and $X_{i_{v}} \in \mathbf{P}_{1}^{\perp}$, so each of these sequences is Cauchy in the metric topology. Lemma 5.29 depends only on the definitions of $\$ 5.1$ and Definition 5.4 and hence applies here. So $\left\|P_{i_{\nu}}\right\| \leqq\left\|T_{i}\right\|$ and $\left\|X_{i_{v}}\right\| \leqq 2\left\|T_{i}\right\|$, and by $[6$, p. 723$]$ each of these sequences is also Cauchy in the strong topology. Hence $P_{i_{v}}$ has a strong limit $P_{i} \in \mathfrak{A}, X_{i_{v}}$ a strong limit $X_{i} \in \mathfrak{A}$, with $P_{i} \in \mathbf{P}_{1}$ and $X_{i} \in \mathbf{P}_{1}^{\perp}$.

By uniqueness of limits, $\theta\left(S_{i}\right)=P_{i}+X_{i}$, and so $\theta^{-1}\left(P_{i}\right)+\theta^{-1}\left(X_{i}\right)=S_{i}$ $\in \mathbf{P}^{\prime \perp}$. Assume that $\theta\left(\mathbf{P}^{\prime}\right)=\mathbf{P}_{1}$. Then $\theta^{-\mathrm{i}}\left(\mathbf{P}_{1}\right)=\mathbf{P}^{\prime}$, so that $\theta^{-1}\left(P_{i}\right) \in \mathbf{P}^{\prime}$, which implies $\theta^{-1}\left(P_{i}\right)=0$. Hence $P_{i}=0,\left[\left[P_{i_{v}}\right]\right] \rightarrow 0$, and $\left[\left[X_{i_{v}}-T_{i}\right]\right] \rightarrow 0$.

Recall that $N_{1}=\left\{K_{1}\right\}$ when $n=1$. Then if $X_{1_{v}}, X_{2_{v}} \in \mathfrak{M}_{q}(q \in \Gamma)$, we have

$$
\begin{aligned}
\left(X_{1_{\nu}} X_{2_{\nu}}\right)^{[q]} & =X_{1_{\nu}}^{|q|} X_{2_{\nu}}^{|q|} \\
& =\left[\sum_{K(r, s)=K_{1}} \alpha_{r s}{ }^{q} E_{r s}\right]\left[\sum_{K(r, s)=K_{1}} \beta_{r s}^{q} E_{r s}\right]=\sum_{K(r, s)=K_{0}} \sigma_{j k}^{q} E_{j k} .
\end{aligned}
$$

So $X_{1_{\nu}} X_{2_{\nu}} \in R\left(\mathscr{C}_{0}\right)=\mathbf{P}_{1}$ for all $\nu$. A familiar computation shows that $\left[\left[X_{1_{\nu}} X_{2_{\nu}}-T_{1} T_{2}\right]\right] \rightarrow 0$, since all operators are uniformly bounded. Hence $T_{1} T_{2}$ is the Hilbert space limit of $X_{1_{\nu}} X_{2_{\nu}} \in \mathbf{P}_{1}$. Since we also have $T_{1} T_{2}$ 
$=\theta\left(S_{1}\right) \theta\left(S_{2}\right) \in \mathfrak{A},\left[6\right.$, p. 728] assures us that $T_{1} T_{2} \in \mathbf{P}_{1}$. However, $T_{1} T_{2}$ $=\theta\left(S_{1} S_{2}\right)=\theta\left(S_{3}\right)=X_{3} \in \mathbf{P}_{1}^{\perp}$, which is a contradiction.

Therefore we cannot have $\theta\left(\mathbf{P}^{\prime}\right)=\mathbf{P}_{1}$. Now if there were a ${ }^{*}$-automorphism $\theta$ such that $\theta\left(\mathbf{R}^{\prime}\right)=\mathbf{R}_{1}$, then we would have $\theta\left(\mathbf{P}^{\prime}\right)=\theta\left[\mathfrak{N}\left(\mathbf{R}^{\prime}\right)\right]=\mathfrak{N}\left[\theta\left(\mathbf{R}^{\prime}\right)\right]$ $=\mathbf{P}_{1}$. But this is again a contradiction, so such $\mathbf{a}^{*}$-automorphism cannot exist.

We have thus shown that the invariant $L$ is not sufficient to determine a complete classification of the semi-regular subalgebras of $\mathscr{U}$.

\section{BiblOGRAPHY}

1. J. Dixmier, Les algèbres d'opérateures dans l'espace Hilbertien, Gauthier-Villars, Paris, 1957. 2. , Sous-anneaux abeliens maximaux dans les facteurs de type fini, Ann. of Math. (2) 59 (1957), 279-286.

3. I. Kaplansky, A theorem on rings of operators, Pacific J. Math. 1 (1951), 227-232.

4. F. I. Mautner, Unitary representations of locally compact groups, Ann. of Math. (2) 51 (1950), 1-25.

5. F. J. Murray and J. von Neumann, On rings of operators, Ann. of Math. (2) 37 (1936), 116-229. 6. , On rings of operators. IV, Ann. of Math. (2) 44 (1943), 716-808.

7. L. Pukánszky, On maximal abelian subrings of factors of type $\mathrm{II}_{1}$, Canad. J. Math. 12 (1960), 289-296.

8. I. E. Segal, Irreducible representations of operator algebras, Bull. Amer. Math. Soc. 53 (1947), 73-88.

9. J. von Neumann, On rings of operators. Reduction theory, Ann. of Math. (2) 50 (1949), 401-485.

Massachusetts Institute of Technology, Cambridge, Massachusettrts 